\title{
Water Dynamics Around Proteins: T- and R-States of Hemoglobin and Melittin
}

\author{
Marco Pezzella, ${ }^{\dagger}$ Krystel El Hage ${ }^{\dagger, \perp}$ Michiel J.M. Niesen, ${ }^{\ddagger}$ Sucheol Shin,, \\ Adam P. Willard, ${ }^{*, \ddagger}$ Markus Meuwly, ${ }^{*, \dagger}$ and Martin Karplus ${ }^{*, \S}$ \\ $\dagger$ Department of Chemistry, University of Basel, Klingelbergstrasse 80, CH-4056 Basel, \\ Switzerland \\ $\ddagger$ Department of Chemistry, MIT, USA \\ IDepartment of Chemistry, University of Texas at Austin, USA \\ $\S$ Department of Chemistry, Harvard University, USA \\ ||Laboratoire de Chimie Biophysique, ISIS, Université Louis Pasteur, 67000 Strasbourg, \\ France \\ $\perp$ SABNP, Univ. Evry, INSERM U1204, Université Paris-Saclay, 91025 Evry, France \\ E-mail: awillard@mit.edu; m.meuwly@unibas.ch; marci@tammy.harvard.edu
}

June 29, 2020

\begin{abstract}
The water dynamics, as characterized by the local hydrophobicity ( $\mathrm{LH})$, is investigated for tetrameric hemoglobin and dimeric melittin. For the $T_{0}$ to $R_{0}$ transition in $\mathrm{Hb}$ it is found that $\mathrm{LH}$ provides additional molecular-level insight into the Perutz mechanism, i.e., the breaking and formation of salt bridges at the $\alpha_{1} / \beta_{2}$ and $\alpha_{2} / \beta_{1}$ interface is accompanied by changes in LH. For Hb in cubic water boxes with $90 \AA$ and $120 \AA$ edge length it is observed that following a decrease in LH as a consequence of
\end{abstract}


reduced water density or change of water orientation at the protein/water interface the $\alpha / \beta$ interfaces are destabilized; this is a hallmark of the Perutz stereochemical model for the $\mathrm{T}$ to $\mathrm{R}$ transition in $\mathrm{Hb}$. The present work thus provides a dynamical view of the classical structural model relevant to the molecular foundations of $\mathrm{Hb}$ function. For dimeric melittin, earlier results by Cheng and Rossky [Nature, 1998, 392, 696699] are confirmed and interpreted on the basis of LH from simulations in which the protein structure is frozen. For the flexible melittin dimer the changes in the local hydration can be as much as $30 \%$ than for the rigid dimer, reflecting the fact that protein and water dynamics are coupled.

\section{Introduction}

Hemoglobin is one of the most widely studied proteins due to its essential role in transporting oxygen from the lungs to the tissues. The two most important structural states of this protein are the deoxy structure $\left(\mathrm{T}_{0}\right)$, which is stable when no ligand is bound to the heme-iron, and the oxy structure $\left(R_{4}\right)$, which is stable when each of the four heme groups have a ligand, such as oxygen, bound to them. The state with the quaternary structure of $\mathrm{R}_{4}$, but with no heme-bound ligands is the $\mathrm{R}_{0}$ state. Despite strong experimental evidence that $\mathrm{T}_{0}$ is significantly more stable than $\mathrm{R}_{0}$, with an equilibrium constant of $K_{\frac{T_{0}}{R_{0}}}=6.7 \times 10^{5}, 1$ molecular dynamics (MD) simulations appear to indicate that the $\mathrm{R}_{0}$ state is more stable. Specifically, simulations have found that when hemoglobin is initialized in the $\mathrm{T}_{0}$ state it undergoes a spontaneous transition into the $\mathrm{R}_{0}$ state on sub- $\mu$ s time scales. ${ }^{213}$ Understanding the origins of this discrepancy between the measured and simulated relative stabilities of the $\mathrm{R}_{0}$ and $\mathrm{T}_{0}$ states is essential to establishing the reliability of simulation-based studies of Hemoglobin and other large biomolecules.

In a recent simulation study, it was found that the $T_{0} \rightarrow R_{0}$ transition rate depends sensitively on the size of the simulation cell. ${ }^{4}$ Specifically, simulations of hemoglobin initialized 
in the $\mathrm{T}_{0}$ state and placed in a periodically replicated cubic solvent box with side length of $75 \AA, 90 \AA$, and $120 \AA$, underwent transition towards the R-state structure after $130 \mathrm{~ns}, 480$ ns, and 630 ns, respectively. Furthermore, in a simulation box with side-length of $150 \AA$, hemoglobin remained in the $\mathrm{T}_{0}$ state for the entirety of a $1.2 \mu \mathrm{s}$ simulation. The extrapolated trend in these findings implies that $\mathrm{T}_{0}$ is the thermodynamically stable state in this largest simulation cell for which the diffusional dynamics of the environment are correctly captured. The results also suggested that such a large box is required for the hydrophobic effect, which stabilizes the $T_{0}$ tetramer, to be manifested. While the statistical significance of this con-

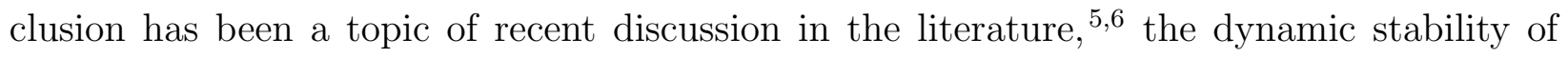
the $\mathrm{T}_{0}$ state exhibits a clear systematic dependence on the size of the solvent box. Further analysis is required to provide conclusive evidence of the role of the hydrophobic effect and to reveal the mechanistic origin of the dependence of the thermodynamic stability of the $\mathrm{T}_{0}$ state on the simulation box size. In this study we specifically address the role of system size variations in solvent dynamics.

The present work addresses the system size question by analyzing the molecular structure of the hydration layers surrounding tetrameric hemoglobin $(\mathrm{Hb})$ and dimeric melittin. The particular focus is on whether there are characteristic changes in local hydration that accompany global transitions involving reorientation of the subunits - i.e. the decay of the T-state for $\mathrm{Hb}$ and the reconfiguration of the helices in melittin - and whether and how these changes are effected by the size of the solvent box. Extending the study to the melittin dimer, which is much smaller than $\mathrm{Hb}$, provides information about the generality of this analysis. In addition, melittin was also studied previously as an example for hydrophobic hydration. ${ }^{7}$ Melittin is a small, 26-amino acid protein found in honeybee venom that crystallizes as a tetramer, consisting of two dimers, related by a two-fold symmetry axis. ${ }^{[19}$ Previous work has characterized the behaviour of the hydrophobic binding surface of melittin and the solvent exposed surface residues. ${ }^{7}$ While these surface residues are characterized by a well-defined 
orientation of the water molecules, water molecules in the hydrophobic regions are more dynamical, exploring different water configurations. Here, similar simulations with a frozen melittin dimer in different box sizes are carried out and analyzed. In addition, the protein is also allowed to move freely which provides information about the solvent-solute coupling which has not been considered before..$^{7}$

The analysis is based on a recently developed method of characterizing the hydrophobicity of a surface based on a statistical analysis of the configurational geometries of interfacial water molecules.10 This method, described in more detail in the "Analysis of Aqueous Interfacial Structure" section, generates an order parameter, $\delta \lambda_{\text {phob }}$, which quantifies the statistical similarity of sampled water configurations to those that occur at equilibrium near an ideal hydrophobic surface. When applied to water configurations sampled from a particular nanoscale region of a protein surface, $\delta \lambda_{\text {phob }}$ can be interpreted as a local measure of hydrophobicity and thus be extended to map the spatial and temporal variations of a protein's solvation shell.

In the following section the simulations and computational methods are described. Then, in the Results section, analyses and interpretations of protein hydration structure are presented. Results for hemoglobin are described first, including analysis of previous simulations in different simulation box sizes. Results for melittin are described second. Finally, conclusions are drawn.

\section{Computational Methods}

\section{Molecular Dynamics Simulations}

Simulations of Hemoglobin (Hb): The Hb-simulations (for the sequence see Figure S1) 
were described previously, $\underline{4}$ and only the necessary points without technical details are reported here. The molecular dynamics trajectories were run in cubic water boxes with box lengths $90 \AA, 120 \AA$, and $150 \AA$ which are analyzed in the following. Each simulation was run for $1 \mu$ s or longer and for selected box sizes, additional repeat simulations were carried out. ${ }^{[6}$ The trajectories analyzed in the present work are those from Ref. ${ }^{[4}$ and the reader is referred to that manuscript for additional details on the production runs.

Simulations of Melittin: MD simulations of the melittin dimer were carried out using CHARMM ${ }^{11}$ c45a1 and the CHARMM36 force field. ${ }^{12}$ The TIP3P water model was used, the same as that used for $\mathrm{Hb}$. The dimer structure (PDB:2MLT) ${ }^{13}$ was used as the starting structure. It was solvated in a cubic water box of length $51.051 \AA$ (4066 water molecules) In addition, simulations with a box length of $60 \AA$ were performed to assess whether, in analogy to $\mathrm{Hb}$, there were effects of increased solvent box sizes on the stability, dynamics and water structuring of melittin dimer. A $16 \AA$ cut-off was applied with a Particle Mesh Ewald scheme ${ }^{14}$ and a 1 fs time step was used in the MD simulations. Although more physically realistic fixed point charge water models exist (e.g. TIP4P), the use of TIP3P here is mandatory for consistency because the CHARMM force field was parametrized with it. It is certainly of interest to include water polarization in corresponding simulations, $\frac{15}{15}$ but such a study is beyond the scope of the present work.

The following protocol was used. Two steps of minimization were performed: 50 steps with the Steepest Descent algorithm, followed by 50 steps with the Newton-Raphson algorithm. The system was then heated and equilibrated using the velocity Verlet algorithm $\frac{16}{16}$ for 25 ps with a Nose Hoover ${ }^{17}$ thermostat at $300 \mathrm{~K}$. This was followed by a $100 \mathrm{~ns}$ NVT production simulation, for which coordinates were recorded every 1 ps. In a first set of simulations, the protein dimer was fixed and only the solvent water was allowed to move. This allows direct comparison with the work of Cheng and Rossky. ${ }^{[7}$ In a separate set of $100 \mathrm{~ns}$ simulations 
the protein was allowed to move and only bonds involving hydrogen atoms molecules were constrained using SHAKE. $\underline{18}$

\section{Analysis of Aqueous Interfacial Structure}

The hydration structure of the simulated proteins was characterized following a recently developed computational method. 10 This method is based on the concept that deformations in water's collective interfacial molecular structure encode information about the details of surface-water interactions. $\frac{19}{19}$ These deformations are quantified in terms of the probability distribution of molecular configurations, as specified by the three-dimensional vector, $\vec{\kappa}=\left(a, \cos \theta_{\mathrm{OH}_{1}}, \cos \theta_{\mathrm{OH}_{2}}\right)$, where $a$ is the distance of the oxygen atom position to the nearest point on the instantaneous water interface, as defined in Ref., 20 and $\theta_{\mathrm{OH}_{1}}$ and $\theta_{\mathrm{OH}_{2}}$ are the angles between the water $\mathrm{OH}$ bonds and the interface normal.

Here, this method is used to compute the time dependent quantity, $\delta \lambda_{\text {phob }}^{(r)}(t)$, which describes the local hydrophobicity $(\mathrm{LH})$ of residue $r$, at time $t$. More specifically, $\delta \lambda_{\text {phob }}^{(r)}(t)=\lambda_{\text {phob }}^{(r)}(t)-$ $\left\langle\lambda_{\text {phob }}\right\rangle_{0}$, where,

$$
\lambda_{\text {phob }}^{(r)}(t)=-\frac{1}{\sum_{a=1}^{N_{a}(r)} N_{w}(t ; a)} \sum_{a=1}^{N_{a}(r)} \sum_{i=1}^{N_{w}(t ; a)} \ln \left[\frac{P\left(\vec{\kappa}^{(i)}(t) \mid \text { phob }\right)}{P\left(\vec{\kappa}^{(i)}(t) \mid \mathrm{bulk}\right)}\right] .
$$

Here the summation over $N_{a}(r)$ is over the atoms in residue $r$ and the summation over $N_{w}(t ; a)$ is over the water molecules within a cut-off of $6 \AA$ of atom $a$ at time $t$, and $\vec{\kappa}^{(i)}(t)$ denotes the configuration of the $i$ th molecule in this population. $P(\vec{\kappa} \mid$ phob $)$ is the probability to find configuration $\vec{\kappa}$ at an ideal hydrophobic surface and $P(\vec{\kappa} \mid$ bulk $)$ is the probability to find that same configuration in the isotropic environment of the bulk liquid. As described in Ref. 10, these reference distributions were obtained by sampling the orientational distribution of water at an ideal planar hydrophobic silica surface and the bulk liquid, respectively. The 
quantity $\left\langle\lambda_{\text {phob }}\right\rangle_{0}$ is the equilibrium value of $\lambda_{\text {phob }}$ for configurational populations sampled from the ideal hydrophobic reference system.

Values of $\delta \lambda_{\text {phob }}^{(r)}$ close to zero indicate that water near residue $r$ exhibits orientations that correspond to those found at an ideal hydrophobic surface. Hydrophilic surfaces interact with interfacial water molecules and lead to configurational distributions that differ from that of an ideal hydrophobic surface. These differences are typically reflected as values of $\delta \lambda_{\text {phob }}^{(r)}>0$, with larger differences giving rise to larger positive deviations in $\delta \lambda_{\text {phob. Val- }}^{(r)}$ ues of $\delta \lambda_{\text {phob }}(r) \geq 0.5$ are used as indicative of hydrophilicity. For the number of unique water configurations used to compute $\delta \lambda_{\text {phob }}^{(r)}$ here, fluctuations of $\delta \lambda_{\text {phob }}^{(r)}$ are expected to fall within $-0.24 \leq \delta \lambda_{\text {phob }}^{(r)} \leq 0.27$ (95\% confidence interval) at the hydrophobic reference system, making sustained values of $\lambda_{\text {phob }}^{(r)} \geq 0.5$ highly indicative of local hydrophilicity. The fluctuations in $\delta \lambda_{\text {phob }}^{(r)}$ as a function of time provide information about changes in the local solvation environment.

\section{Results}

\section{Hydration Dynamics around $\mathbf{T}_{0^{-}}$and $\mathbf{R}_{0^{-}}$State Hemoglobin}

Figure 1 (top) illustrates the structure of $\mathrm{Hb}$ for the sequences of the $\alpha$ and $\beta$ chains) with the $\mathrm{C}_{\alpha}$ atoms of the residues analyzed specifically shown as van der Waals spheres. The first set of residues we study are the ones in Perutz' stereochemical model, ${ }^{21}$ which are involved in the salt bridges and the $\alpha / \beta$ shearing motion (Table 1). The shearing motion involves a change in the H-bonds at the $\alpha_{1} / \beta_{2}$ interface. In the $\mathrm{T}_{0}$ structure, the side chain of Thr $41 \alpha_{1}$ occupies a notch formed by the main chain of Val98 $\beta_{2}$ and a hydrogen bond is present between Tyr42 $\alpha_{1}$ and Asp99 $\beta_{2}$. After the transition to the $\mathrm{R}_{4}$ state, the same notch is occupied instead by Thr $38 \alpha_{1}$ and the previous hydrogen bond is substituted by one between Asp94 $\alpha_{1}$ 
and Asn $102 \beta_{2}$. The same conformational change occurs at the $\alpha_{2} / \beta_{1}$ interface.

Other R-state structures, including $R_{2}, R_{2}$ and $R_{3}$, also exist and present intermediate states between $\mathrm{T}_{0}$ and $\mathrm{R}_{4}$. The difference between all $\mathrm{Hb}$ forms emerges from differences in the position of the $\beta_{2}$-subunit relative to the $\alpha_{1}$-subunit at the switch region. $\frac{22}{22}$ This can be shown by superimposing the $\mathrm{C}_{\alpha}$ atoms of the $\alpha_{1} \beta_{1}$ dimers and computing the RMSD for the $\alpha_{2} \beta_{2}$ dimer with the $\mathrm{T}_{0} \mathrm{C}_{\alpha}$ atoms structure as a reference (Figure 1 , top panel) for all $\mathrm{Hb}$ forms extracted from crystal structures and for the $\mathrm{T}_{0} \mathrm{Hb}$ structure simulated in different water box sizes. The same applies to superimposing the $\mathrm{C}_{\alpha}$ atoms of $\alpha_{2} \beta_{1}$ dimers and computing the RMSD of the nonsuperimposed regions $\left(\alpha_{1} \beta_{2}\right.$ dimer, and also on the $\alpha$ carbons) with the $\mathrm{T}_{0}$ structure as a reference point (Figure 1, left bottom panel). And as a measure of quaternary variation, the complete $\alpha_{1} \beta_{1} \alpha_{2} \beta_{2}$ tetramer was superimposed on the $\mathrm{C}_{\alpha}$ atoms (Figure $\mathrm{S} 2$ ) 3rd panel from top). It is found that these different RMSD results follow the same trends when comparing different $\mathrm{R}$ forms and box sizes. $\mathrm{R}_{3}$ shows the most shift and is closest to $T_{0}$, followed by $R_{2}$ and lastly by the $R_{2}$ and $R_{3}$ structures.

Figure 1 also shows that the large quaternary structural difference between the $\mathrm{T}$ and $\mathrm{R}$ forms is accompanied by significant changes in the $\alpha_{1}-\alpha_{2}$ and $\beta_{1}-\beta_{2}$ iron-iron distances; they are reduced in the R-states, most notably for the $\mathrm{R}_{3}$ structure (right panels). This movement of the subunits has a large effect on the interdimer interface (as observed in the interaction distances reported in Figure $\mathrm{S} 3$ ) and thus on the central water cavity relative to the $\mathrm{T}_{0}$ structure. There is also the change in the $\mathrm{C}_{\alpha}-\mathrm{C}_{\alpha}$ distance between His $146 \beta_{1}$ and His146 $\beta_{2}$

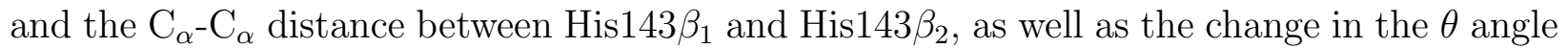
between the two planes containing His $146 \beta_{1}-\mathrm{Fe} \beta_{1}-\mathrm{Fe} \alpha_{1}$ and His146 $\beta_{2}-\mathrm{Fe} \beta_{2}-\mathrm{Fe} \alpha_{2}$. The angle between the $\alpha_{1} \beta_{1}$ and $\alpha_{2} \beta_{2}$ subunits is smaller in all $\mathrm{R}$-forms compared to the $\mathrm{T}$ structure (Figure S2, bottom panel). These values explain the shorter distances between His146 $\beta_{1}$ and His $146 \beta_{2}$, and between His143 $\beta_{1}$ and His143 $\beta_{2}$ reported in Figure S2 (first two panels from 


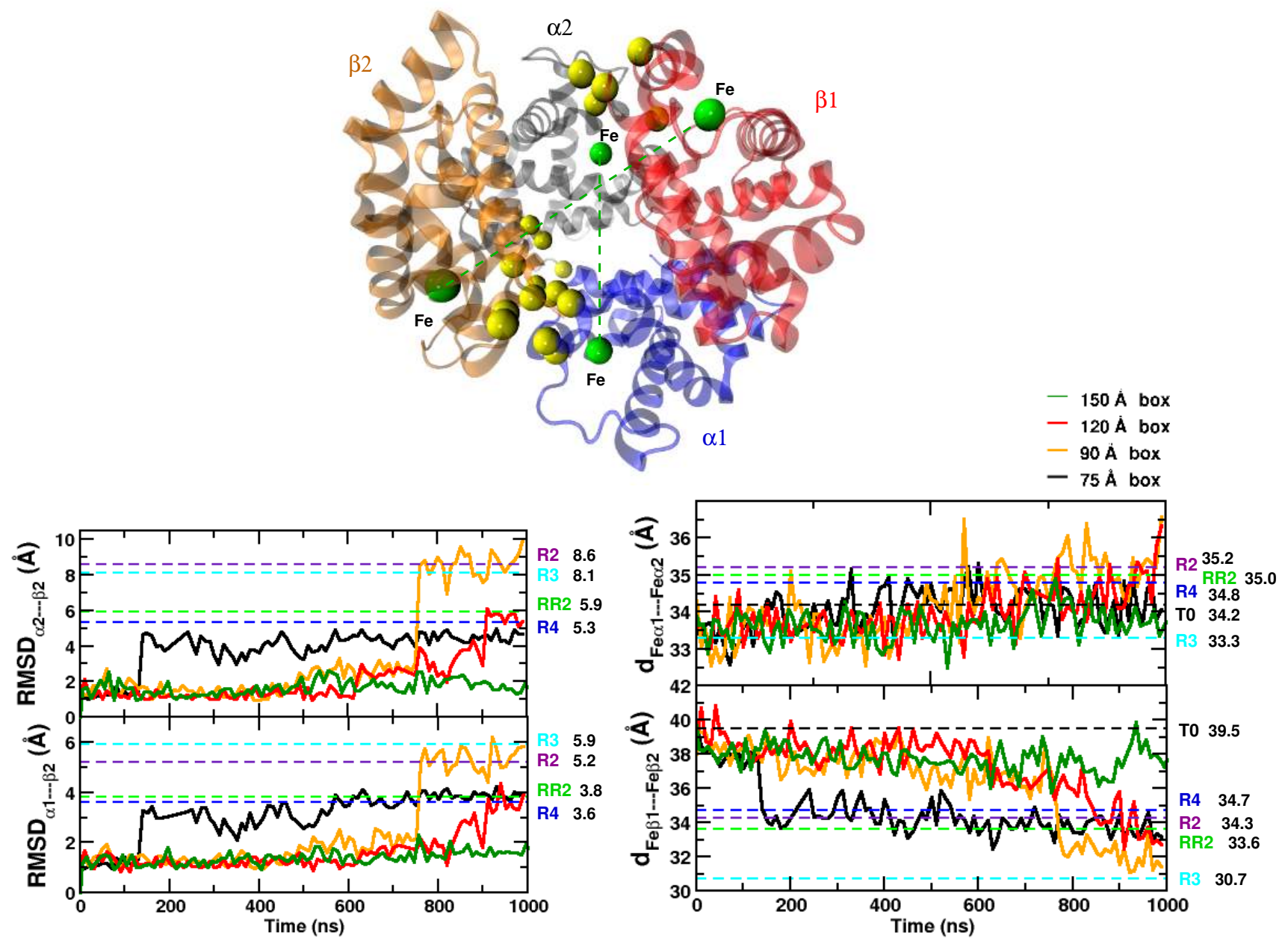

Figure 1: Top: Representation of $\mathrm{Hb}$ with the $\mathrm{C}_{\alpha}$ atoms of the residues relevant to Perutz' stereochemical mechanism shown as yellow spheres. The iron atoms (green spheres) are connected with green dashed lines, indicating the distances reported. Blue, red, grey, and gold ribbon structures for $\alpha_{1}, \beta_{1}, \alpha_{2}$, and $\beta_{2}$ subunits of Hb. Bottom: Quaternary structure differences of $\mathrm{Hb}$ structures found in the simulations, based on the structural comparison of the $\alpha_{1} \beta_{1}$ and $\alpha_{2} \beta_{2}$ subunits. Black, gold, red, and green traces for simulations in the $75 \AA$, $90 \AA, 120 \AA$, and $150 \AA$ boxes, respectively. Left panels: (top) RMSD of the nonsuperimposed $\alpha_{2} \beta_{2}$ subunit after superimposing the $\alpha_{1} \beta_{1}$ subunit $\left(\mathrm{C}_{\alpha}\right.$ carbon atoms were used for both superposition and RMSD calculation); (bottom) RMSD of the nonsuperimposed $\alpha_{1} \beta_{2}$ subunit after superimposing the $\alpha_{2} \beta_{1}$ subunit. Right panels: Iron-Iron distances between the $\alpha$ s and $\beta_{\mathrm{s}}$ of each subunit. Horizontal dashed lines indicate the corresponding values from all known $\mathrm{Hb}$ structures $\left(\mathrm{T}_{0}, \mathrm{R}_{2}, \mathrm{RR}_{2}, \mathrm{R}_{3}\right.$ and $\left.\mathrm{R}_{4}\right)$. 
top). Hence, the $\beta$-cleft entrance to the central water cavity is narrowed (compared to the $\mathrm{T}_{0}$ structure with the largest central cavity) and this leads to less water entering the central cavity. The decrease in the number of water molecules in the central cavity was noted in our previous paper ${ }^{4}$ where water molecules present in the central cylinder for the different box sizes were counted (see Figures 5-figure supplement 3 and 4 in Ref. ${ }^{4}$ ).

Local structural changes around His146 resulting from differences in the position of the $\beta_{2^{-}}$ subunit relative to the $\alpha_{1}$-subunit are also observed (Figure S3). In all R forms compared to the T structure, the water-mediated contact (His146 $\beta$ ) COO-OC(Pro37 $\alpha$ ) and the salt bridges between (His146 $\beta$ )COO-NZ(Lys40 $\alpha$ ) and (His146 $\beta)$ NE2-COO(Asp94 $\beta$ ) are absent. Further, the salt bridge missing in the $\mathrm{T}_{0}$ form between (His146 $\beta$ ) COO and $\mathrm{NE}(\mathrm{His} 2 \beta)$ is observed only in the $\mathrm{R}_{4}$ form.

Specific H-bonds at the $\alpha_{1} \beta_{2}$ dimer interface involved in the shearing motion were also analyzed (Figure S4). First, the hydrogen bond between Thr38 $\alpha_{1}$ and His $97 \beta_{2}$, present in the $R_{3}$ structure but missing in the $R_{2}$ and $R_{2}$ intermediate structures, was sampled in our simulations. Second, the hydrogen bond between Tyr $42 \alpha_{1}$ and Asp99 $\beta_{2}$ present only in the $\mathrm{T}_{0}$ structure and absent in all R-forms was observed for the stable $\mathrm{T}_{0}$ state simulation (150 $\AA$ box) and was absent in all boxes with transitions. Finally, the hydrogen bond between Arg92 $\alpha_{1}$ and Gln39 $\beta_{2}$ or Glu43 $\beta_{2}$ present in $\mathrm{RR}_{2}$ and missing in all other states was observed.

The conformational differences between the $\mathrm{T}$ and $\mathrm{R}$ states affect the hydration environment in a manner that can be related to $\delta \lambda_{\text {phob }}^{(r)}$. Based on the results of previous simulations, $\frac{4}{4}$ the set of residues for which $\delta \lambda_{\text {phob }}$ changes most across the transitions was selected. Figure 2 (top panel) reports the $\mathrm{C}_{\alpha}$ His146 $\beta_{1}-$ His146 $\beta_{2}$ separation, which serves as an indicator of the T-to- $\mathrm{R}$ transition for the simulations in the 90, 120, and $150 \AA$ boxes. For the simulations in the two smaller boxes, three red transitions are evident between the $\mathrm{T}_{0}$-state (at early times) 
and the $\mathrm{R}_{0}$-state (at late times, see Figure 1 in Ref. ${ }^{[4}$ ), as indicated by the red dashed lines in Figure 2. Structural changes are accompanied by changes in the number of hydration waters. For the simulation in the largest $(150 \AA)$ box, for which no transition occurs, the $\mathrm{C}_{\alpha}$ His146 $\beta_{1}-$ His $146 \beta_{2}$ separation is constant and the average hydration is larger than 0.95 (see bottom row in Figure 2).

(a) Results for Hb $90 \AA$ box: Local hydrophobicity (LH) for residues identified as the Perutz stereochemical model (see Table 1). The LH analysis for the $1 \mu \mathrm{s}$ simulation is carried out with a time resolution of $0.5 \mathrm{~ns}$. A cut-off of $6 \AA$ from the protein is chosen to distinguish between interfacial and bulk water. The structural transitions for the $90 \AA$ box occur at $t=470 \mathrm{~ns}, t=770$, and $t=891 \mathrm{~ns}$, as indicated by the distance $r_{\text {His } 146}$ between the $\mathrm{C}_{\alpha}$ atoms of the two His146 residues in the $\beta_{1}$ and $\beta_{2}$ chains. The total number of interfacial water is found to correlate with this distance (see bottom row of Figure 22). Whenever the distance between the two His146 residues (see reference ${ }^{\sqrt{4}}$ ) decreases abruptly (as indicated by the red dashed lines), the relative number of water molecules $r_{w}$ within the $6 \AA$ cut-off increases. The value of $r_{w}=N_{\text {wat }} / N_{\max }$ was determined as the instantaneous number $N_{\text {wat }}$ of water molecules for a specific snapshot and the maximum $N_{\max }$ encountered along the entire trajectory.

To obtain more detailed information, $\delta \lambda_{\text {phob }}(t)$ was analyzed for the residues listed in Table 1. see Figure 3. For certain residues, structural transitions (at $t=470 \mathrm{~ns}, t=770 \mathrm{~ns}$, and $t=891 \mathrm{~ns}$ ) are accompanied by abrupt rather than by gradual changes in local hydrophobicity of individual residues. Examples include Val98 $\beta_{1}$, Thr $41 \alpha_{1}$, or Asp $94 \alpha_{1}$. By contrast, Tyr $42 \alpha_{1}$ shows a gradual decrease in $\delta \lambda_{\text {phob }}$ over most of the $1 \mu$ s simulation. There are also changes in LH away from overall structural transitions, e.g. for Val98 $\beta_{2}$, Asp99 $\beta_{2}$, and Asn $102 \beta_{2}$ at 200 ns, further discussed below. Except for Val98 $\beta_{1}$ all residues that show a substantial decrease in their hydrophilic $\left(\delta \lambda_{\text {phob }} \sim 0.5\right)$ versus hydrophobic $\left(\delta \lambda_{\text {phob }} \sim 0\right)$ 


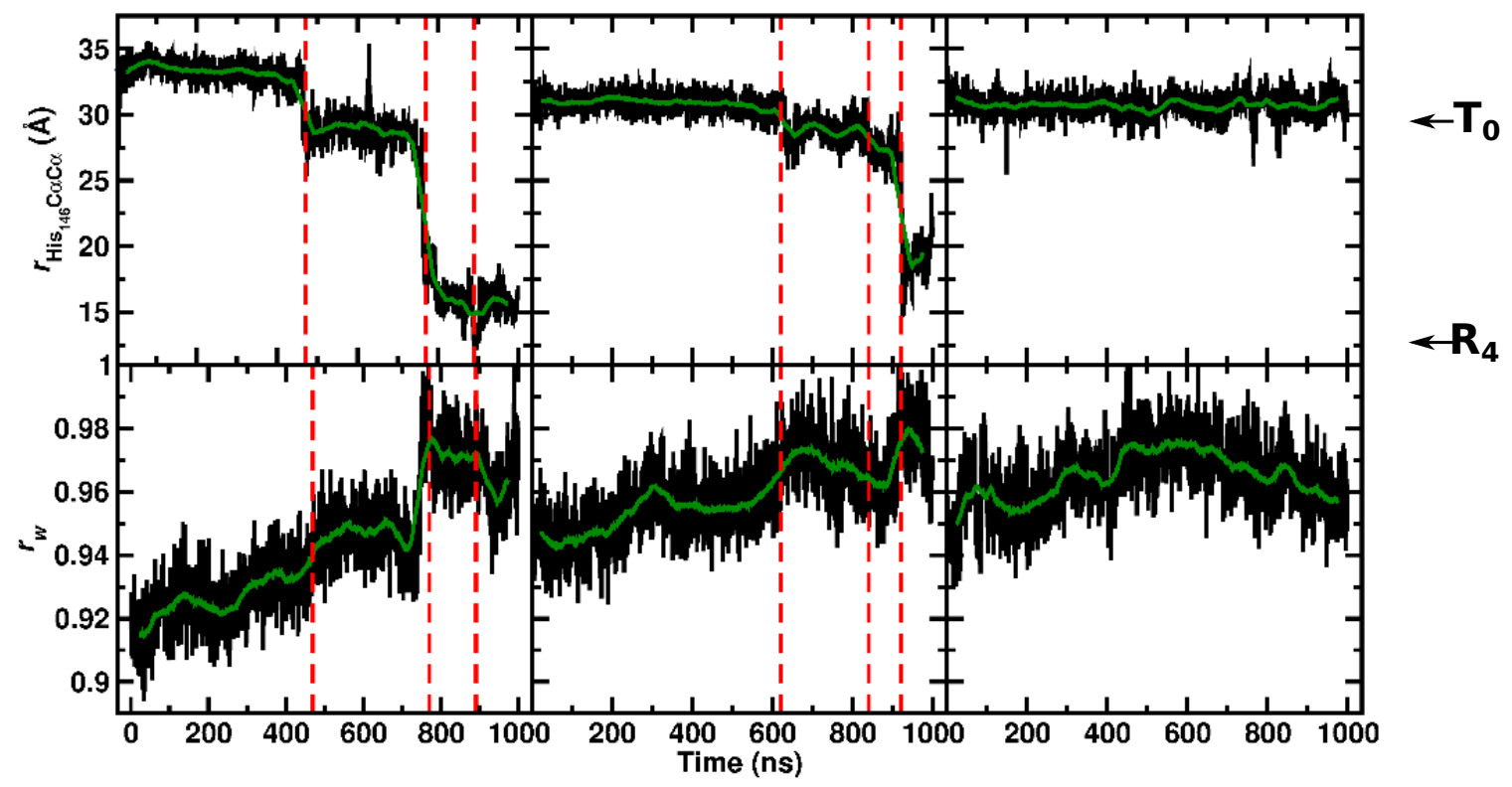

Figure 2: Top: The $\mathrm{C}_{\alpha} \operatorname{His}_{146} \beta_{1}-\mathrm{His}_{146} \beta_{2}$ separation as a function of time for the 90, 120 and $150 \AA$ box from left to right. Raw data in black and running averages over 50 ns in green. The arrows on the right-hand side indicate the $\operatorname{His}_{146} \beta_{1}-\mathrm{His}_{146} \beta_{2}$ separation $\left(r_{\mathrm{His} 146, \mathrm{C} \alpha \mathrm{C} \alpha}\right)$ in the 2DN2 $\left(\mathrm{T}_{0}\right)$ and 2DN3 $\left(\mathrm{R}_{4}\right)$ crystal structures. ${ }^{23}$ Bottom: The hydration (" $\mathrm{H}_{2} \mathrm{O}$ ratio") of the entire protein, expressed in terms of $r_{w}=N_{\text {wat }} / N_{\max }$. The vertical red dashed lines indicate the transition times for the three steps observed in the $90 \AA$ and $120 \AA$ boxes (see Figure 1B of reference. $\underline{4}$

character $\left[\operatorname{Thr} 41 \alpha_{1}, \operatorname{Tyr} 42 \alpha_{1}\right]$ or an increase $\left[\operatorname{Thr} 38 \alpha_{1}, \operatorname{Asp} 94 \alpha_{1}\right.$, Asp99 $\left.\beta_{2}\right]$ are at the $\alpha_{1} / \beta_{2}$ interface. This suggests that the decay for the $90 \AA$ box is triggered primarily by the hydration around residues that are involved in the $\alpha_{1} / \beta_{2}$ contacts.

Based on the data in Figure 3, the $\mathrm{T}_{0} \rightarrow \mathrm{R}_{0}$ transition in the $90 \AA$ box is accompanied by significant changes in the hydration environment at certain locations around the $\alpha_{1} / \beta_{2}$ contact. This observation is consistent with Perutz' conclusion. We quote, "[..]Where is the force that changes the quaternary structure applied[..]The evidence is overwhelmingly in favor of the contacts $\alpha_{1} \beta_{2}[.$.$] ". 21$ The significant changes in hydration around the $\alpha_{1} \beta_{2}$ contact suggest the possibility that the $\mathrm{T}_{0} \rightarrow \mathrm{R}_{0}$ transition is driven by solvent thermodynamics. 
Table 1: The residues of $\mathrm{Hb}$ for which the local hydrophobicity $\delta \lambda_{\text {phob }}$ is analyzed from Perutz' stereochemical model. ${ }^{21}$ For each residue its involvement in specific contacts is reported.

\begin{tabular}{|c|c|}
\hline Residue & Role in the protein \\
\hline $\operatorname{Arg} 141 \alpha_{1}$ & $\alpha$ C-terminal salt bridge \\
\hline Vall $\alpha_{2}$ & $\alpha$ C-terminal salt bridge \\
\hline Asp $126 \alpha_{2}$ & $\alpha$ C-terminal salt bridge \\
\hline Lys $127 \alpha_{2}$ & $\alpha$ C-terminal salt bridge \\
\hline Tyr140 $\alpha_{1}$ & $\alpha$ proximity to the $\mathrm{C}$-terminal residue \\
\hline His146 $\beta_{1}$ & $\beta$ C-terminal salt bridge \\
\hline Lys $40 \alpha_{2}$ & $\beta$ C-terminal salt bridge \\
\hline Asp94 $\beta_{1}$ & $\beta$ C-terminal salt bridge \\
\hline Tyr $145 \beta_{1}$ & $\beta$ salt bridge involved in His146 $\beta_{1}$ motion \\
\hline Val98 $\beta_{1}$ & $\beta$ salt bridge involved in His $146 \beta_{1}$ motion \\
\hline Thr38 $\alpha_{1}$ & $\alpha_{1}-\beta_{2}$ shearing \\
\hline Thr $41 \alpha_{1}$ & $\alpha_{1}-\beta_{2}$ shearing \\
\hline $\operatorname{Tyr} 42 \alpha_{1}$ & $\alpha_{1}-\beta_{2}$ shearing \\
\hline Asp94 $\alpha_{1}$ & $\alpha_{1}-\beta_{2}$ shearing \\
\hline Cys $93 \beta_{2}$ & $\alpha_{1}-\beta_{2}$ shearing \\
\hline Val98 $\beta_{2}$ & $\alpha_{1}-\beta_{2}$ shearing \\
\hline Asn $102 \beta_{2}$ & $\alpha_{1}-\beta_{2}$ shearing \\
\hline Asp99 $\beta_{2}$ & $\alpha_{1}-\beta_{2}$ shearing \\
\hline
\end{tabular}

(b) Results for Hb $120 \AA$ box: For the simulation in the $120 \AA$ box most of the residues involved in the salt bridges, like those in the $90 \AA$ box, show only minor variations in $\Delta\left\langle\delta \lambda_{\text {phob }}\right\rangle$ except that of Tyr145 $\beta_{1}$ and Val98 $\beta_{1}$ which have the largest variations along the trajectory (see Figure $4 \mathrm{~B}$ ). It is found that the LHs of all other residues in Figures $3 \mathrm{~A}, \mathrm{~B}$ and 4A, B behave similarly in the simulations of the $90 \AA$ and $120 \AA$ boxes. For Val98 $\beta_{1}$, instead of decaying to $\Delta\left\langle\delta \lambda_{\text {phob }}\right\rangle \approx 0$ as in the simulation of the $90 \AA$ box, the value of $\Delta\left\langle\delta \lambda_{\text {phob }}\right\rangle$ in the $120 \AA$ box remains at or above 0.5 throughout the entire simulation. Hence, the $\mathrm{T}_{0}$ $\rightarrow \mathrm{R}_{0}$ transition is again mainly associated with motion at the $\alpha_{1} / \beta_{2}$ interface (see Figure 4 C,D). An example of a transition that follows the mechanism described by Perutz ${ }^{21}$ is the transition at 620 ns at the $\alpha_{2} / \beta_{1}$ interface, where the cleavage of the Tyr $42 \alpha_{2}$ and Asp99 $\beta_{1}$ salt bridge is clearly visible (see Figure S4 middle panel and Figure S5). 
Several of the residues at the $\alpha_{1} / \beta_{2}$ interface show pronounced changes in local hydrophobicity that coincide with structural transitions (Figures S3 and S4). However, a few residues in Figure 4D also show LH changes that are not necessarily linked directly to a tertiary structural change ("step"); they are Val98 $\beta_{2}$, Asp99 $\beta_{2}$, and Asn102 $\beta_{2}$ at around 500 ns, further discussed below. For the transitions at 620 ns and 840 ns there is again a clear change in LH for Thr38 $\alpha_{1}$, Thr41 $\alpha_{1}$, and Asp94 $\alpha_{1}$, the most pronounced of them involving Thr38 $\alpha_{1}$. These observations also indicate that the nature of the transition at $470 \mathrm{~ns}$ (step1) in the 90 $\AA$ and at 620 ns (step1) in the $120 \AA$ box is different and may be explained by the transition to different intermediate R-forms (see next paragraph).

For the decaying structures in the $90 \AA$ and $120 \AA$ boxes the following is observed. In the $90 \AA$ box, a transition from $\mathrm{T}_{0}$ to $\mathrm{R}_{3}$ starts at $470 \mathrm{~ns}$ (step1), where the His146-His146 separation drops from $31 \AA$ to $25 \AA$ bringing the Hb structure closer to $\mathrm{R}_{3}(22 \AA$, Figure $\mathrm{S} 2$, first panel). This $\mathrm{T}_{0}$ to $\mathrm{R}_{3}$ transition is completed at $780 \mathrm{~ns}$ (step2, Figure $\mathrm{S} 2$, the presence of the $\mathrm{R}_{3}$ structure at $780 \mathrm{~ns}$ is marked in all the panels by a cyan dashed line). At $\sim 880$ ns (step3) a next transition from $R_{3}$ to $R_{2}$ occurs (the $R_{2}$ structure is marked by a violet dashed line in Figure $\mathrm{S} 2$ ). For the rest of the simulation until $1 \mu \mathrm{s}$, the $\mathrm{RR}_{2}$ and $\mathrm{R}_{4}$ states are sampled (Figure $\mathrm{S} 2, \mathrm{RR}_{2}$ and $\mathrm{R}_{4}$ states are indicated by a green dashed line and a blue arrow, respectively). Conversely, in the $120 \AA$ box, at step1 at $620 \mathrm{~ns}$ a $\mathrm{T}_{0}$ to $\mathrm{R}_{2}$ transition starts by decaying to an unknown intermediate. It is continued at $840 \mathrm{~ns}$ (step2) to bring the structure closer to $R_{2}$. The transition to $R_{2}$ is completed by 920 ns (step3, Figure $S 2$ ), the presence of the $R_{2}$ structure at $920 \mathrm{~ns}$ is indicated in all the panels by a vertical violet dashed line).

(c) Results for Hb $150 \AA$ box: For the $150 \AA$ box the previous MD simulations did not find a structural transition. ${ }^{4}$ The values of $\delta \lambda_{\text {phob }}$ for all residues involved in the salt bridges (Figure 5, columns A and B) do not deviate significantly from their average value. 


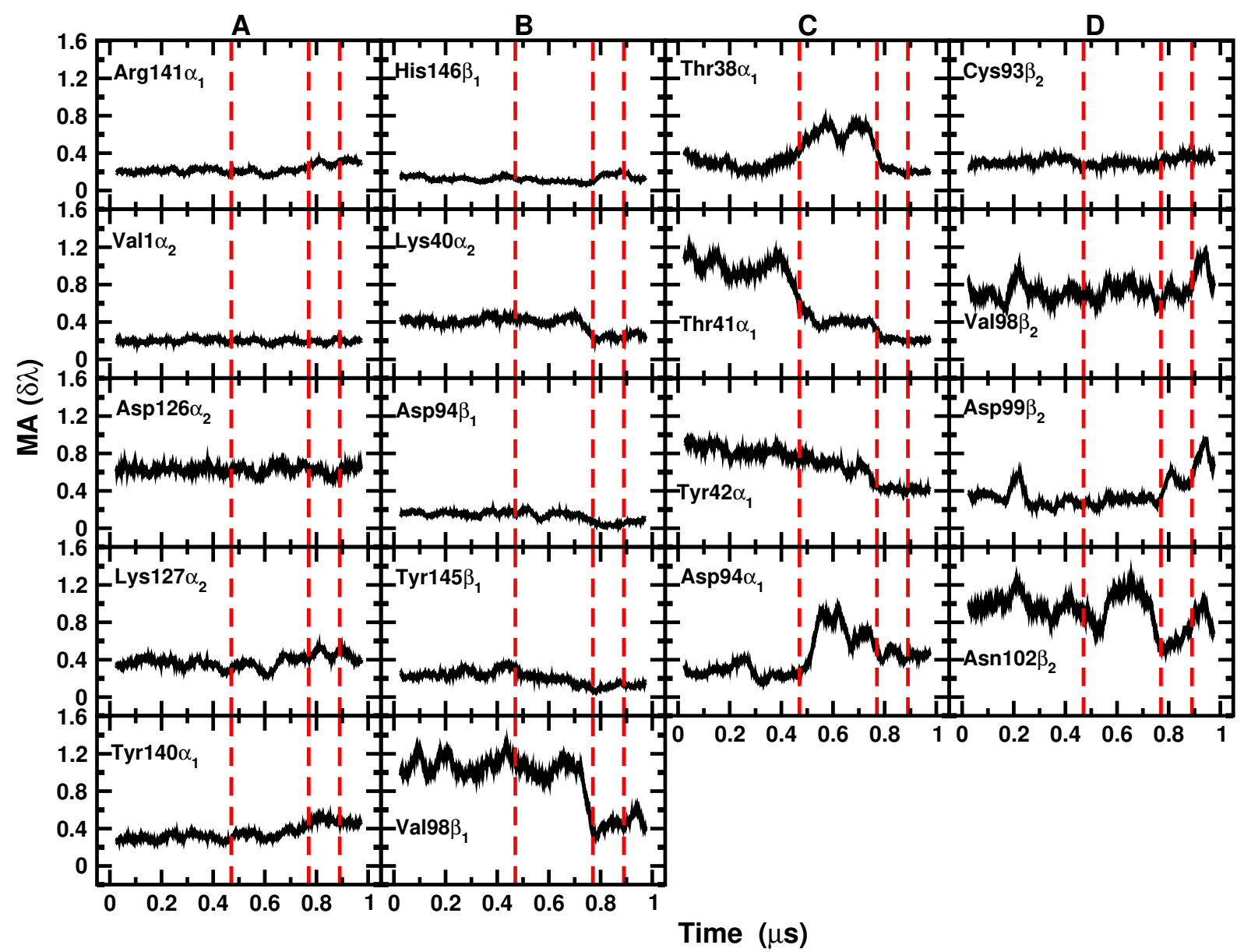

Figure 3: Local hydrophobicity for the simulation in the $90 \AA$ box. Moving average (MA) over $50 \mathrm{~ns}$ of $\delta \lambda_{\text {phob }}$ as a function of time for residues involved in the C-terminal salt bridges 21 (column A), additional salt bridges (column B) and the $\alpha_{1} \beta_{2}$ (columns C and D); see Table 1 .

The amplitude of the fluctuations are typically smaller than for the simulations in the $90 \AA$ and $120 \AA$ boxes. For the residues at the $\alpha_{1} \beta_{2}$ interface there are variations for $\operatorname{Thr} 38 \alpha_{1}$, Thr $41 \alpha_{1}$, Tyr42 $\alpha_{1}$, and Asp94 $\alpha_{1}$ (see Figure 5).

The clearest difference between the simulation in the $150 \AA$ and the two smaller boxes is the behaviour for residues Val98 $\beta_{2}$, Asp99 $\beta_{2}$, and Asn102 $\beta_{2}$. As an example, the water occupation around Val98 $\beta_{2}$ is analyzed by computing the radial distribution function $g(r)$ between the $\mathrm{C}_{\alpha}$ of the residue and the surrounding hydration water for different parts of the 


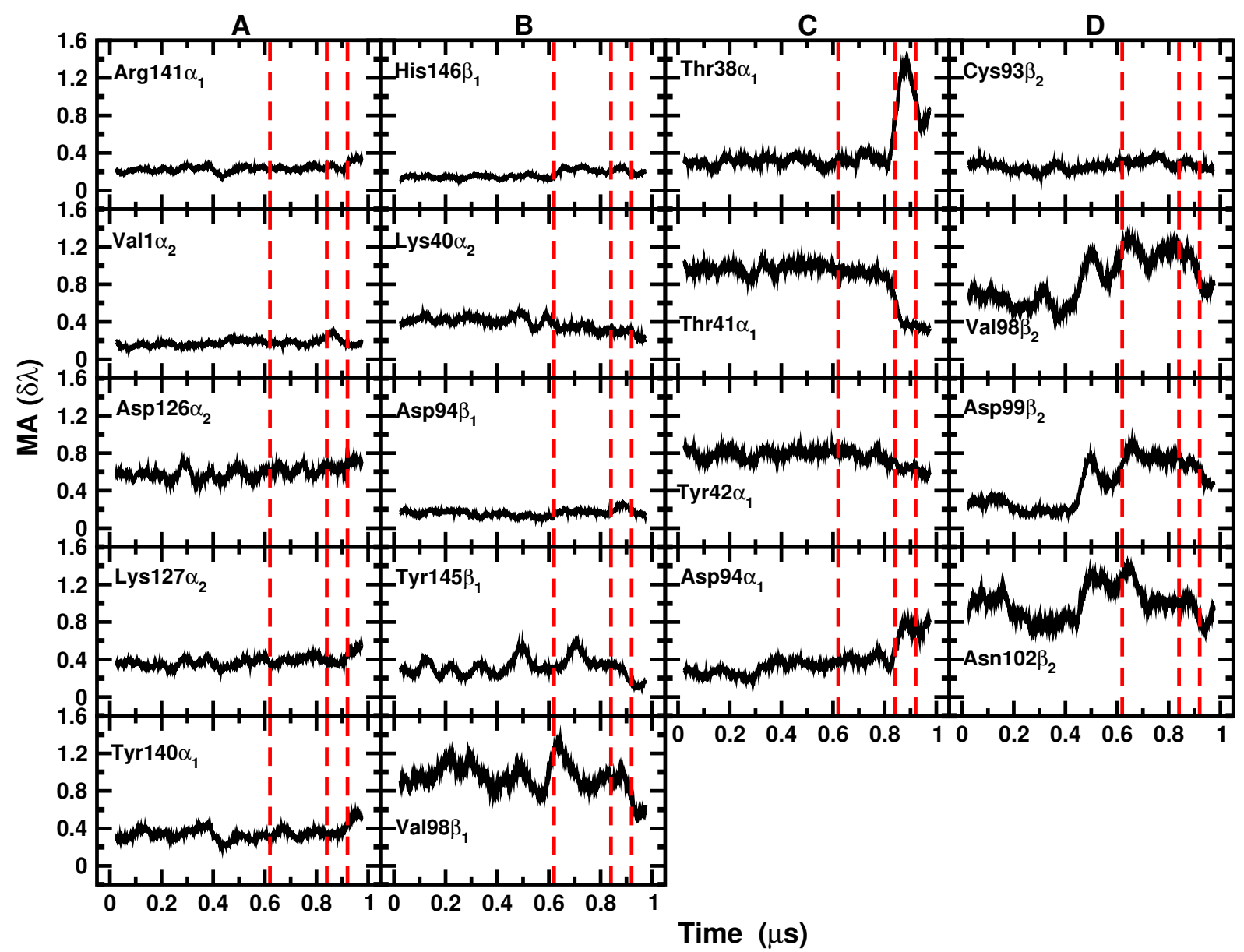

Figure 4: Local hydrophobicity for the simulation in the $120 \AA$ box. Moving average [MA $(\delta \lambda)]$ over 50 ns of $\delta \lambda_{\text {phob }}$ as a function of time for residues involved in the C-terminal salt bridges $^{21}$ (column A), additional salt bridges (column B) and the $\alpha_{1} \beta_{2}$ shearing motion (columns C and D); see Table1. The presence of the $\mathrm{R}_{2}$ structure at $920 \mathrm{~ns}$ is indicated in all the panels by a vertical violet dashed line.

trajectory. The radial distribution functions $g(r)$ in Figure S6 show that they are close in shape to one another but differ in magnitude for the early phase of the trajectory in the 120 $\AA$ and $150 \AA$ box. They change in shape after the transition at $840 \mathrm{~ns}$ in the smaller of the two boxes. A pronounced signature in LH is also found in the $120 \AA$ and $150 \AA$ boxes for Thr38 $\alpha_{1}$ between 800 and 900 ns. The signatures in LH for the $150 \AA$ box can be related to formation of a Thr $38 \alpha_{1}-$ Asp99 $\beta_{2}$ salt bridge (Figure S7). Breaking and reforming of salt bridges involving Val98 $\beta_{2}$, Asp99 $\beta_{2}$, and Asn102 $\beta_{2}$ is also responsible for the sharp increase in LH around these three residues in the $90 \AA$ box around 200 ns, see Figures 3D and S8. 


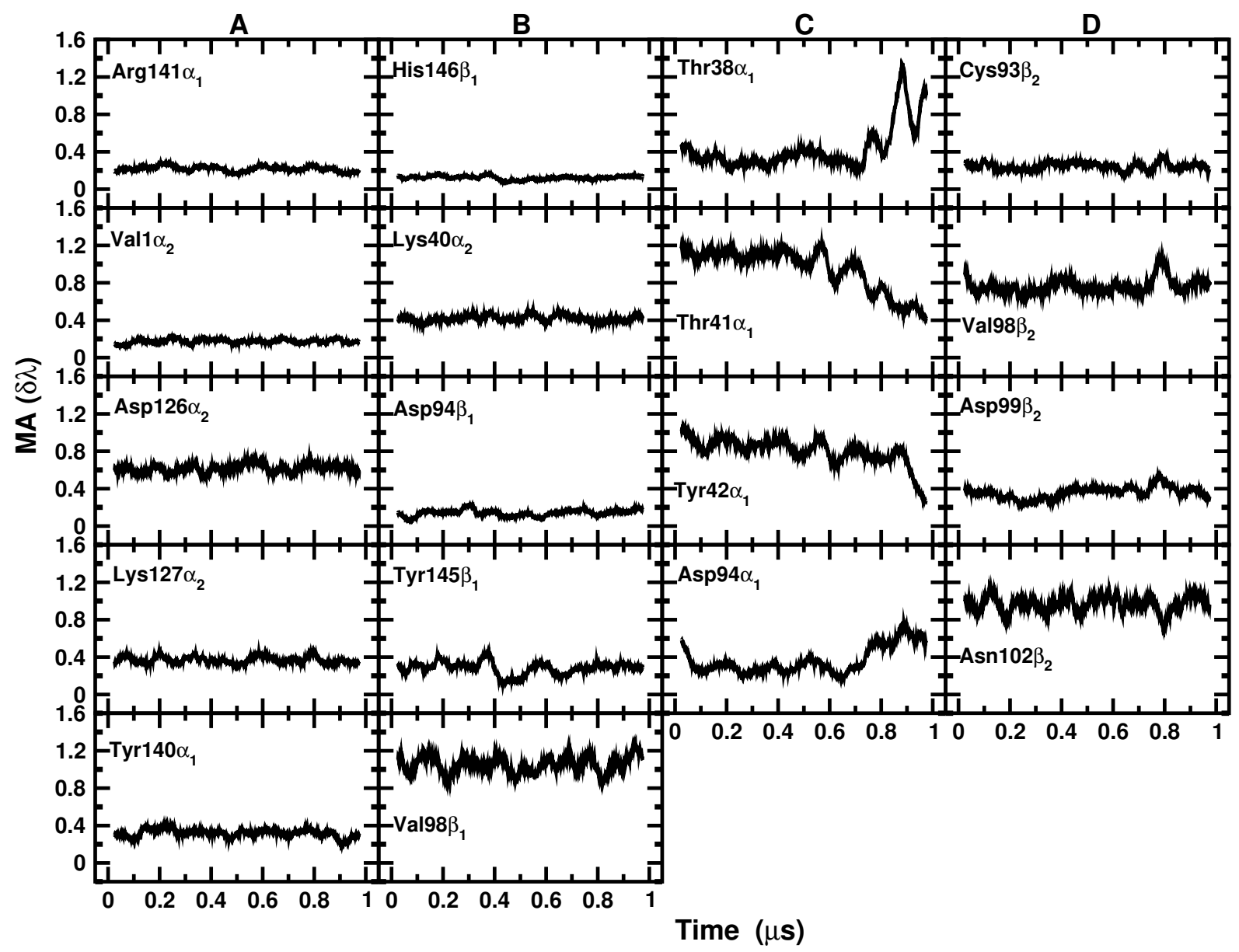

Figure 5: Local hydrophobicity for the simulation of the $150 \AA$ box. It shows moving averages over $50 \mathrm{~ns}$ of $\delta \lambda_{\text {phob }}$ as a function of time for residues involved in the C-terminal salt bridges ${ }^{21}$ (column A), additional salt bridges (column B) and the $\alpha_{1} \beta_{2}$ shearing motion (columns $\mathrm{C}$ and $\mathrm{D})$, see Table 1 .

It is notable that the $\mathrm{LH}$ around the three residues already starts to change before the salt bridge actually breaks.

Changes of the LH of each residue can be due to a) internal motion of a residue or b) the influence of neighbouring residues. Both of these are potentially followed by water displace-

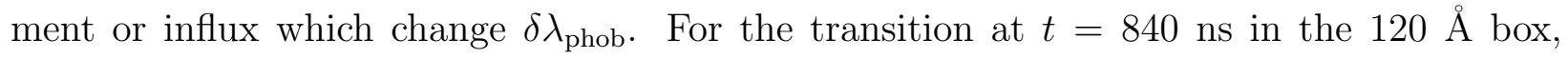
changes in the local water occupation around Val98 $\beta_{2}$ obtained by analysis of radial distribution functions (see Figure S6) do not necessarily lead to changes in LH. The $g(r)$ for the 
time intervals 0 to $620 \mathrm{~ns}$ and $620 \mathrm{~ns}$ to $840 \mathrm{~ns}$ are very similar (red and green lines in Figure S6) while the LH changes from 0.8 at early times to 1.2 after $t \sim 500$ ns, see Figure 4 . The water influx is a consequence of the reconfiguration of the H-bonding network including the Tyr42 $\alpha_{1}-$ Asp99 $\beta_{2}$ salt bridge (see Figure S9) and the rearrangement of the carboxy group of the sidechain of Asp99 $\beta_{2}$ due the rehydration of the side chain. These effects are also mirrored by the Asp99 $\beta_{2}$ carboxy orientation (see dihedral time series reported in Figure S11) which demonstrates that before the transition at $840 \mathrm{~ns}$ the side chain follows a twostate behaviour but after the transition almost free rotation occurs (see also Figure S4). This change is accompanied by increased hydration of the side chain (bottom panel of Figure S11).

Comparing Figures 3 to 5 it is noted that even when $\mathrm{Hb}$ is still in its $\mathrm{T}_{0}$ state (i.e. before $470 \mathrm{~ns}$, the first transition in the $90 \AA$ box), differences in LH, mainly at the $\alpha_{1} / \beta_{2}$ interface are observed. Examples include residues Thr $41 \alpha_{1}$ and Tyr $42 \alpha_{1}$ for which LH oscillates or decreases in the $90 \AA$ box but remains constant in the two larger boxes before 470 ns. The finding that destabilization of the $\alpha_{1} / \beta_{2}$ interface is at the origin of the $T_{0}$ to $R_{0}$ transition is consistent with the Perutz stereochemical model. Conversely, the LH around the C-terminal salt bridge residues is very similar for the simulations in the three different box sizes, except for Val98 $\beta_{1}$ and Tyr145 $\beta_{2}$.

\section{(d) Spatio-temporal analysis based on two-dimensional correlation maps: To bet-} ter understand the coupling of local hydration dynamics and the structural transitions, twodimensional correlation maps were generated which are referred to as local hydrophobicity cross correlation maps (LH-CCMs). Similar to dynamic cross correlation maps (DCCMs) $24 \sqrt[25]{2}$ for residues $i$ and $j$ the quantity

$$
C_{i j}=\frac{\left\langle\Delta \delta \lambda_{\mathrm{phob}}^{(i)} \Delta \delta \lambda_{\mathrm{phob}}^{(j)}\right\rangle}{\sqrt{\left\langle\left(\Delta \delta \lambda_{\mathrm{phob}}^{(i)}\right)^{2}\right\rangle\left\langle\left(\Delta \delta \lambda_{\mathrm{phob}}^{(j)}\right)^{2}\right\rangle}}
$$


was determined for each interval for which $\mathrm{Hb}$ was in a particular conformational state as shown in Figure 1 .

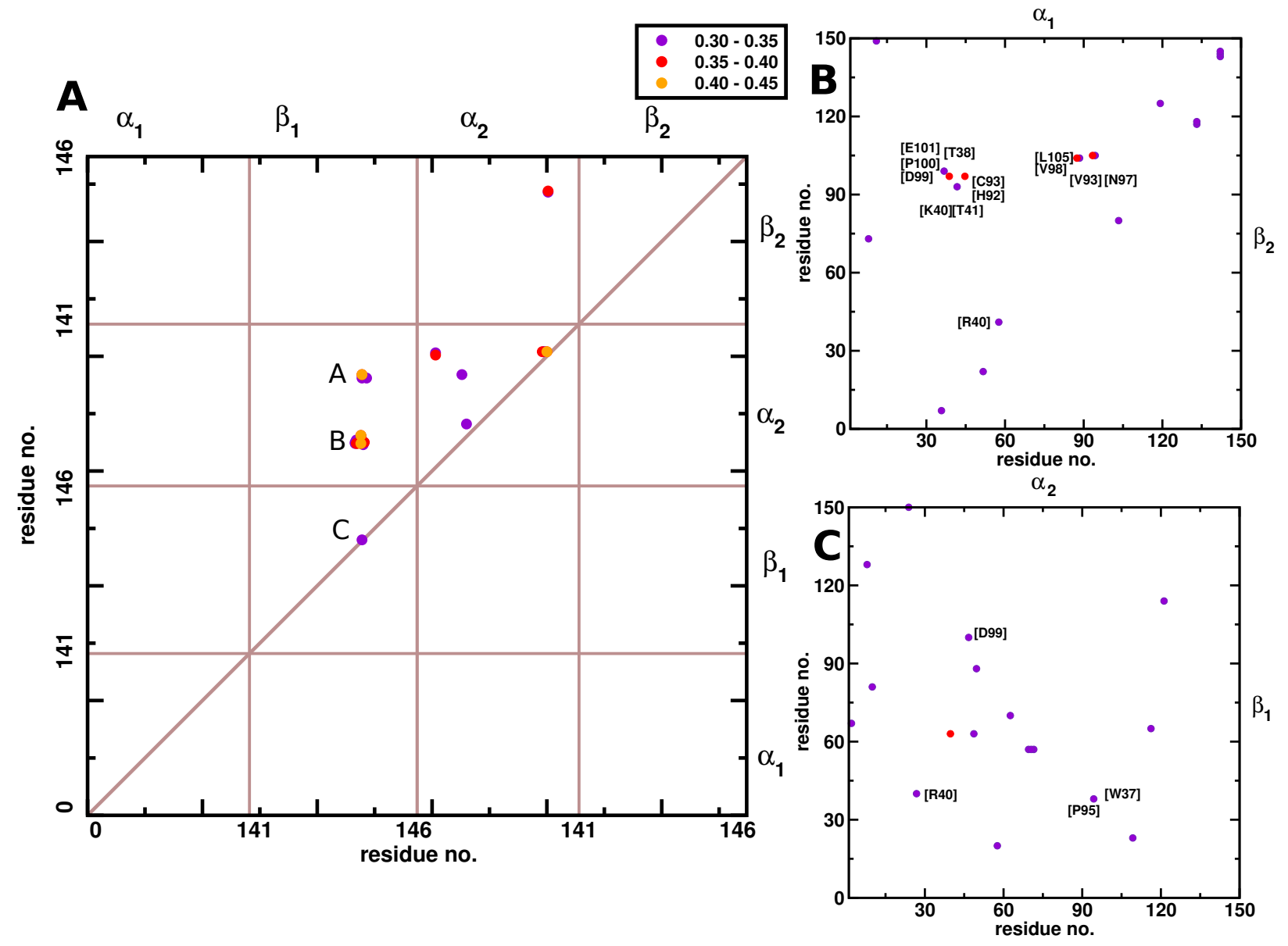

Figure 6: Difference of Local Hydrophobicity $\Delta C_{i j}$ Cross Correlation Map (LH-CCM) for the $120 \AA$ box. Panel A: difference between [0-620] and [620-840], i.e. "transition at 620" for entire protein. Panel B: difference between [620-840] and [840-920], i.e. "transition at 840" for the $\alpha 1 / \beta 2$ interface. Panel C: difference between [620-840] and [840-920], i.e. "transition at 840" for the $\alpha 2 / \beta 1$ interface. The cross correlations for the individual states are given in Figure S12. Only values $\Delta C_{i j} \geq 0.30$ are reported. Color code: $0.30 \leq \Delta C_{i j}<0.35$ (purple), $0.35 \leq \Delta C_{i j}<0.40$ (red), and $0.40 \leq \Delta C_{i j}<0.45$ (orange).

Figure 64 reports the difference between the local hydrophobicity cross correlation maps between time intervals 0 to $620 \mathrm{~ns}$ and 620 to $840 \mathrm{~ns}$ for the $120 \AA$ box for values of $\Delta C_{i j}>0.3$. This map indicates that correlations in LH and their difference can depend on both the sequence and spatial proximity of two residues. The correlation in LH up to 620 ns (i.e. before 
the first transition, see Figure S10) is large $\left(C_{i j}>0.30\right)$ for residues that play an active role in interface transitions between the two subunits (Figure 6 above the diagonal) and for regions that are spatially close (on the diagonal). An example for sequence proximity is the Val98 $\beta_{1}$-Asp99 $\beta_{1}$ region (feature $\mathrm{C}$ in Figure 6A and Figure S9). Changes in LH are a direct consequence of the Asp99 $\beta_{1}$-Tyr $42 \alpha_{2}$ salt bridge cleavage during the transition at 620 ns (see Figure S5) that leads to a change in the orientation of the peptide bond between Val98 $\beta_{1}$-Asp99 $\beta_{1}$ (Figure S9) and corresponding decrease in the LH of both, Asp99 $\beta_{1}$ and Tyr42 $\alpha_{2}$. Examples for spatial proximity of residues are the two clusters (labelled A and B in Figure 6A) that are at the $\alpha_{2} \beta_{1}$ shearing interface. This change in hydrophobicity in one of the two important stabilizing regions of the protein (the other being the $\alpha_{1} / \beta_{2}$ interface), indicates its possible involvement in the destabilization of the $T_{0}$ structure.

A more detailed view of the LH cross correlations for the $\alpha_{1} \beta_{2}$ interface for the $120 \AA$ box is provided in Figure S10 (for LH-CCMs in the $90 \AA$ and $150 \AA$ boxes see Figures S13 and S14). Figure S10 shows the LH cross correlations for residues involved in the $\alpha_{1} / \beta_{2}$ shearing motion up to 620 ns. The clusters (A to E) in Figure S10 involve correlated changes in LH at the $\alpha_{1} / \beta_{2}$ interface, whereas for cluster $\mathrm{F}$ no direct contact is present. In cluster $\mathrm{A}$ the correlation is caused by the $\operatorname{Thr} 41 \alpha_{1}-\operatorname{Arg} 40 \beta_{2}$ salt bridge present before the transition at 620 ns. Cluster B is dominated by the water-mediated Asp $94 \alpha_{1}-\operatorname{Arg} 40 \beta_{2}$ salt bridge before the transition at $620 \mathrm{~ns}$; it is a weak interaction due to the large distance $(\sim 6 \AA)$ between the proton and the anion. After the transition this salt bridge becomes the dominant interaction in which $\operatorname{Arg} 40 \beta_{2}$ is involved. The $\mathrm{C}$ cluster is dominated by the $\pi$-stacking interaction between Tyr $140 \alpha_{1}$ and Trp37 $\beta_{2}$. A weak salt bridge of the Thr38 $\alpha_{1}$ and Asp99 $\beta_{2}$ sidechains with the Thr $41 \alpha_{1}$ sidechain and Asp99 $\beta_{2} \mathrm{NH}$ peptide bond are responsible for the D cluster. The NH peptide bond of Asn $97 \alpha_{1}$ and the Asp99 $\beta_{2}$ side chain lead to cluster E. Overall, this figure provides a dynamic view of the stereochemical model proposed by Perutz. ${ }^{21}$ This is illustrated, for example, by the fact that all clusters (A to F) are extended, rather than the 
usual point-to-point contacts (i.e., the salt bridges) alone.

Next, the transition in the $120 \AA$ box at $840 \mathrm{~ns}$ is discussed from the perspective of the LH-CCMs (see Figure 6 panels B and C). They show the difference between the LH-CCMs for the time intervals [620-840] ns and [840-920] ns, respectively. During the process two salt bridges are broken (Thr41 $\alpha_{1}-\operatorname{Asp} 99 \beta_{2}$ and Thr $41 \alpha_{1}$-Arg $40 \beta_{2}$, which is water mediated) and two new salt bridges are formed (Thr38 $\alpha_{1}-$ Asp99 $\beta_{2}$ and Asp94 $\alpha_{1}-\operatorname{Arg} 40 \beta_{2}$ ) and Asn97 $\alpha_{1}$ Asp99 $\beta_{2}$ continues to show a bimodal behaviour, see Figure S15. It is found that the reformation of these salt bridges between residues involved in the "Perutz mechanism" (Thr38 $\alpha_{1}$, Thr41 $\alpha_{1}$, Asp94 $\alpha_{1}$ and Asp99 $\beta_{2}$ ) is also reflected in the difference cross correlation maps (Figure $6 \mathrm{~B}$ and $\mathrm{C}$ ). They confirm that most of the changes for this transition occur at the $\alpha_{1} / \beta_{2}$ interface. Also, these two panels show that changes in the LH-CCMs are not necessarily symmetric for the $\alpha_{1} / \beta_{2}$ and $\alpha_{2} / \beta_{1}$ interfaces. Such a "dynamical asymmetry" (i.e., it is found in the molecular dynamics simulations) has also been observed for insulin dimer ${ }^{26}$ for which the X-ray structure has $\mathrm{C}_{2}$ symmetry ${ }^{27}$ or is very close to symmetric with only small local deviations from it. 28

As previous results have shown, the relative stability of the $T_{0}$ state depends on the size of the simulation cell. ${ }^{[4}$ Analysis of hydration structure via $\delta \lambda_{\text {phob }}^{(r)}$ has the ability to reveal when and where protein hydration properties differ between differently sized simulation cells. To highlight this point, the statistics and dynamics of $\delta \lambda_{\text {phob }}^{(r)}$ for $\mathrm{Hb}$ in the $\mathrm{T}_{0}$ state in the 90 $\AA$ and $150 \AA$ simulation boxes are compared in Figure 7. It summarizes the values of $\delta \lambda_{\text {phob }}^{(r)}$ over the residues that comprise the $\alpha_{1} / \beta_{2}$ and $\alpha_{2} / \beta_{1}$ interfaces, as a function of simulation time. Most notably, as the conformational transition from the $T_{0}$ state to the $R_{0}$ state progresses, there is a distinct shift towards values of $\delta \lambda_{\text {phob }}^{(r)}$ near zero. This indicates that the interfacial water structure shifts from that observed at a hydrophilic surface towards that observed at a hydrophobic surface (Figure 7A). The shift in interfacial water structure is also 
apparent from the probability distribution, $P\left(\delta \lambda_{\text {phob }}^{(r))}\right)$, for the different simulation box sizes. During the first 500ns of the trajectories the probability distributions overlap (Figure 7B), showing that the interfacial water structure does not differ significantly. However, during the last $500 \mathrm{~ns}$ of the trajectories the probability distribution for the $90 \AA$ simulation box has a significant shift towards lower values of $\delta \lambda_{\text {phob }}^{(r)}$, corresponding to a more hydrophobic character of the interface (Figure 7C).
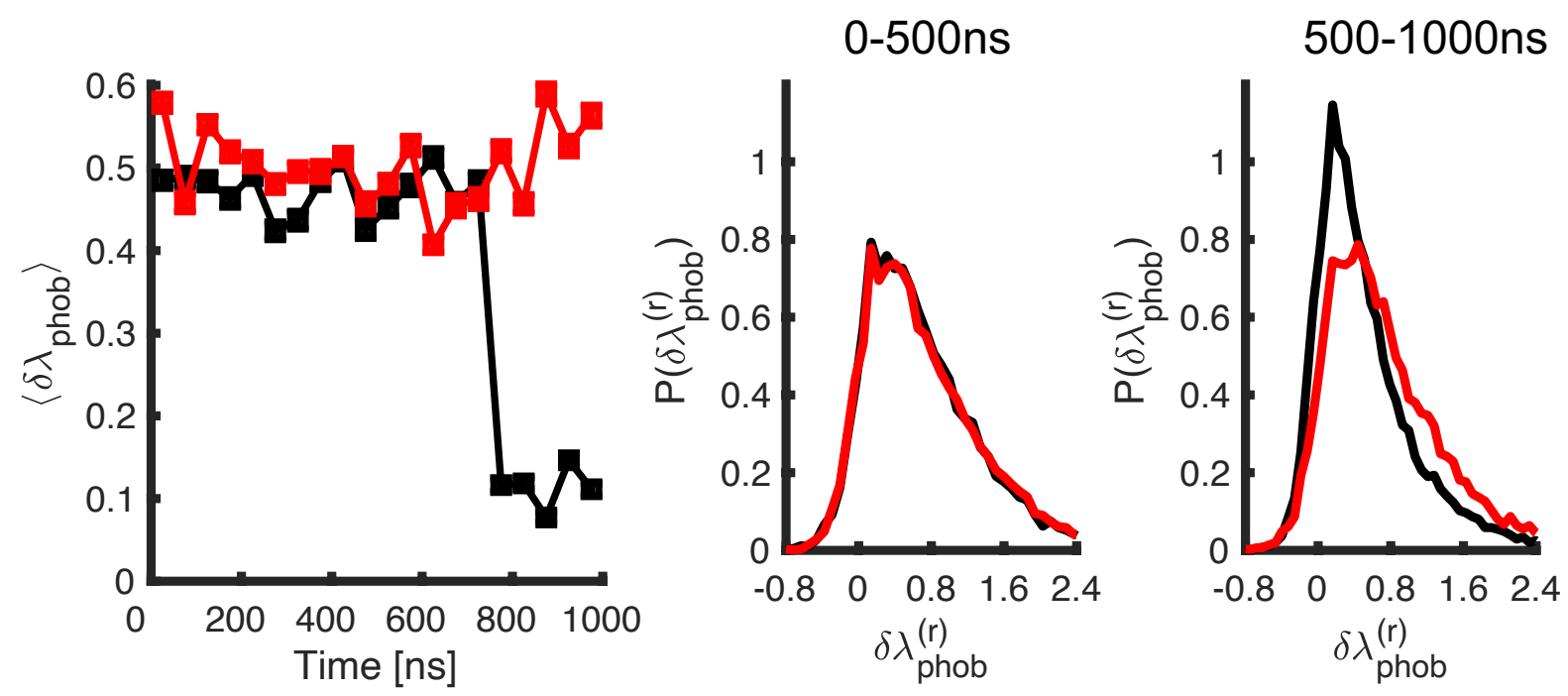

Figure 7: Comparison of the interfacial water structure for residues that are part of the $\alpha_{1} / \beta_{2}$ and $\alpha_{2} / \beta_{1}$ interfaces. The black and red lines correspond to simulations carried out in $90 \AA$ and $150 \AA$ solvent boxes, respectively. The marker indicates the center of a time interval. Panel A: The expectation value of $\delta \lambda_{\text {phob }}^{(r)}$ as a function of time, averaged over $50 \mathrm{~ns}$ time intervals. Panel B: The distribution of $\delta \lambda_{\text {phob }}^{(r)}$ values during the first $500 \mathrm{~ns}$ of the trajectories. Panel C: The distribution of $\delta \lambda_{\text {phob }}^{(r)}$ values during the last $500 \mathrm{~ns}$ of the trajectories.

\section{Hydration Dynamics around Melittin}

As a second example, the analysis of water hydration for $\mathrm{Hb}$ has been extended to melittin. It is a well studied prototype of a protein complex that is stabilized through hydrophobic interactions. Melittin is a small, 26-amino acid protein (for the sequence, see Figure S16) found in honeybee venom that crystallizes as a tetramer, consisting of two dimers (see Fig- 
ure 8 left), related by a two-fold symmetry axis. $\frac{89}{6}$ Cheng and Rossky ${ }^{[7}$ characterized the behaviour of the hydrophobic surface of the melittin dimer and of the surrounding surface residues by simulations in which the structure of the melittin dimer was frozen. They found that in hydrophilic regions the water molecules have a well defined orientation, while in the hydrophobic regions, the waters are more mobile and explore different configurations. ${ }^{7}$ To further explore the hydration dynamics, simulations with a frozen melittin dimer in different box sizes are carried out and analyzed. In additional simulations, the protein was also allowed to move freely. These provide information about the solvent-solute coupling.
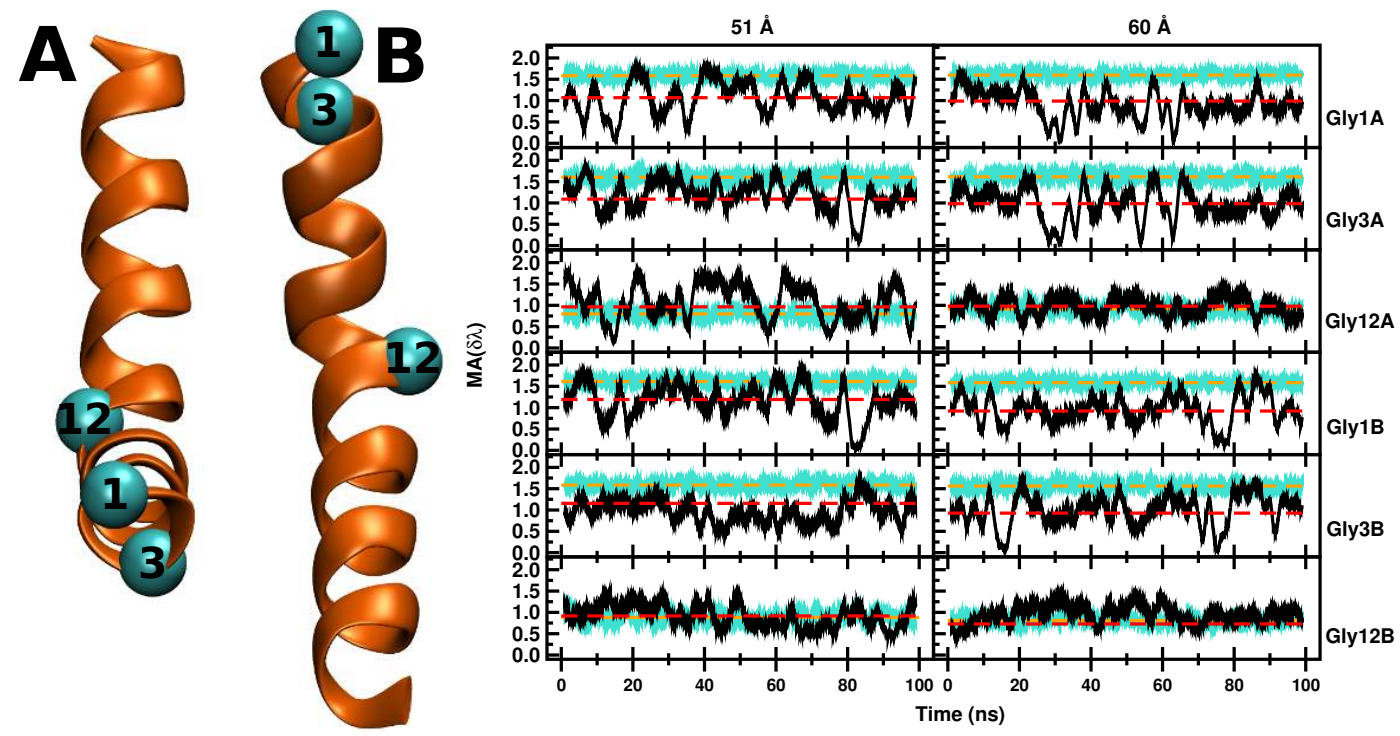

Figure 8: Left: A- and B-helices with the Gly1, Gly3, and Gly12 residues represented as spheres. The A-helix is slightly bent at the C-terminal end for the rigid and flexible simulations. The RMSD of the two chains of the dimer is $1.6 \AA$. Right: Time evolution of $\delta \lambda_{\text {phob }}$ for the 6 glycine residues in the melittin dimer. The results of the simulations for the rigid (cyan) and flexible (black) protein are reported. The orange and red line represent the average $\delta \lambda_{\text {phob }}$ for each residue during the simulation. Gly12 is less hydrophilic than the other Gly residues since it is located in the hydrophobic region of the protein.

It is of interest to analyze whether $\delta \lambda_{\text {phob }}$ encapsulates corresponding information, and whether simulations of water around a rigid melittin dimer, as carried out in Ref., ${ }^{7}$ and around a flexible dimer lead to qualitatively similar results. Since the results for Hb depend on the box size, simulations are also carried out with different box sizes. 


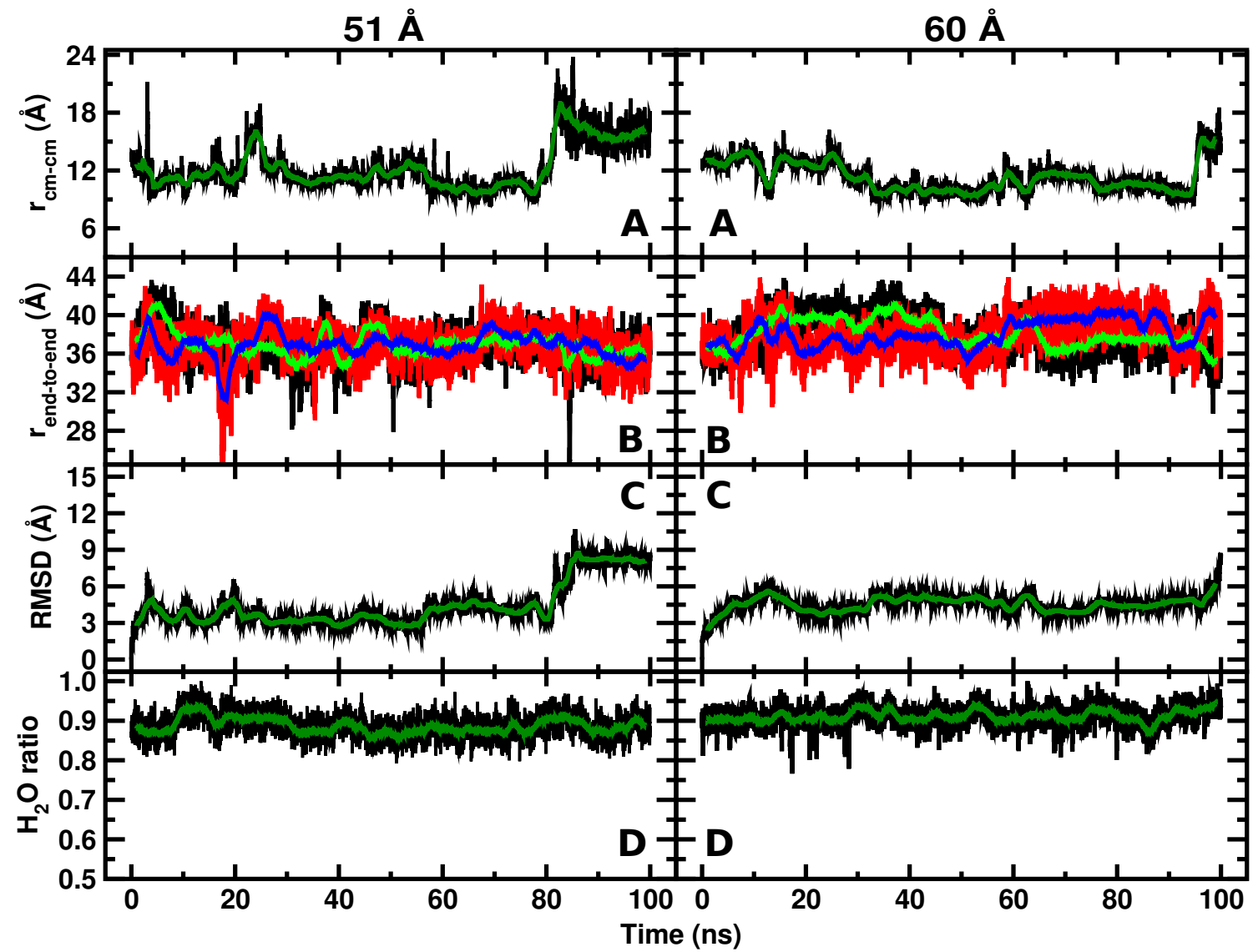

Figure 9: Flexible melittin in the $51 \AA$ and $60 \AA$ boxes. In Panels $\mathrm{A}$ the center-of-mass distance between the two monomers $\left(r_{\mathrm{cm}-\mathrm{cm}}\right)$ is reported as function of time (black: raw data; green: moving average). Around $80 \mathrm{~ns}$, a rearrangement of the two monomers is associated with an increase of $r_{\mathrm{cm}-\mathrm{cm}}$. The chain length $\left(r_{\text {end-to-end }}\right)$ for the two segments (black and red for the raw data for chain A and B, green and blue for their moving average), are illustrated in Panels B. The RMSD with respect to the initial structure of the protein is shown in Panels C. During the time evolution, the number of water surrounding the protein seems not to be influenced by the structural changes as shown in Panels D.

The structural variations together with the overall hydration of the flexible melittin dimer in the different water box sizes are reported in Figure 9, In all simulations the end-to-end separation of the two helices (see Figure $9 \mathrm{~B}$ ) as defined by the $\mathrm{C}_{\alpha}-\mathrm{C}_{\alpha}$ separation of the two terminal residues Gly1 and Gln26 is stable, indicating that the helices (the $\mathrm{H}_{2} \mathrm{O}$ ratio) remain intact. Consequently, the structural transition that occurs after 80 ns in the $51 \AA$ box (Figure 9 C) and appears to occur towards the end of the simulation in the $60 \AA$ box involves 
the dimerization interface. This is confirmed by panel A, which reports an increase of the center-of-mass distance $r_{\mathrm{cm}-\mathrm{cm}}$ between the two helices at the same time as the RMSD in panel C increases. The degree of hydration (Figure 9D) defined as $r_{w}=N_{\text {wat }} / N_{\max }$ remains essentially constant throughout the simulations.

The protein-water interface is analysed using the same methodology as that used for Hb. The Willard-Chandler interface is calculated setting $r_{\text {cut }}=25.0 \AA$ and the likelihood $\left(\delta \lambda_{\text {phob }}\right)$ of the interfacial water with the reference TIP3P water model is determined with a $6 \AA$ cut-off. Figures 8 and S17 show the time evolution of selected residues. Figure 8 shows the temporal evolution, $\delta \lambda_{\text {phob }}$, of glycine residues Gly1, Gly3, and Gly12 for chains A and B for both the rigid and the flexible dimers. The $\delta \lambda_{\text {phob }}$ for the rigid dimer is essentially constant, as expected. The results reported are all averages over 2 ns windows.

Table 2: Average $\delta \lambda_{\text {phob }}$ for the glycine residues for the $100 \mathrm{~ns}$ simulation (see Figure 8).

\begin{tabular}{l|r|r|r|r} 
Residue & $51 \AA$ flexible & $51 \AA$ rigid & $60 \AA$ flexible & $60 \AA$ rigid \\
\hline $1 \mathrm{~A}$ & 1.068 & 1.583 & 0.989 & 1.598 \\
$3 \mathrm{~A}$ & 1.090 & 1.604 & 0.983 & 1.616 \\
$12 \mathrm{~A}$ & 0.965 & 0.882 & 0.979 & 0.945 \\
$1 \mathrm{~B}$ & 1.192 & 1.614 & 0.921 & 1.587 \\
$3 \mathrm{~B}$ & 1.156 & 1.584 & 0.927 & 1.568 \\
$12 \mathrm{~B}$ & 0.917 & 0.799 & 0.968 & 0.826
\end{tabular}

For the rigid monomer (blue traces in Figure 8) the LH is constant along the entire $100 \mathrm{~ns}$ simulation for both box sizes and the averages differ by $10 \%$ at most (Gly12A). For the flexible dimer (black traces) the instantaneous LH fluctuates around well-defined average values except for Gly12B which has a slight increase in its dynamics during the early phase of the simulation, particularly in the $60 \AA$ box. In the simulation in both box sizes the amplitude of LH fluctuates between 0 and 1.6, i.e. between being hydrophobic and hydrophilic. Since Gly is an aliphatic/neutral residue, the changing hydrophilicity must be a consequence of its embedding along the peptide chain and the water structuring around it. Overall, it is 
found that Gly12A and 12B, which are near the middle of the helix, are less hydrophilic (see Figure 8 and Table 2) than Gly1 and Gly3, which are positioned at or near the terminus. This difference is more pronounced for the rigid dimer.

Figure S17 shows the LH for the residues investigated by Cheng and Rossky ${ }^{7}$ for the $51 \AA$ box while those for the $60 \AA$ box are given in Figure S18. The average $\delta \lambda_{\text {phob }}$ are neutral or hydrophilic. Good qualitative agreement with $\operatorname{Ref}^{[7}$ is found for residues Val8A (hydrophilic), Leu9A, Ile13A, Ile13B (residues with a decreasing level of hydrophobicity), and Ile20B.

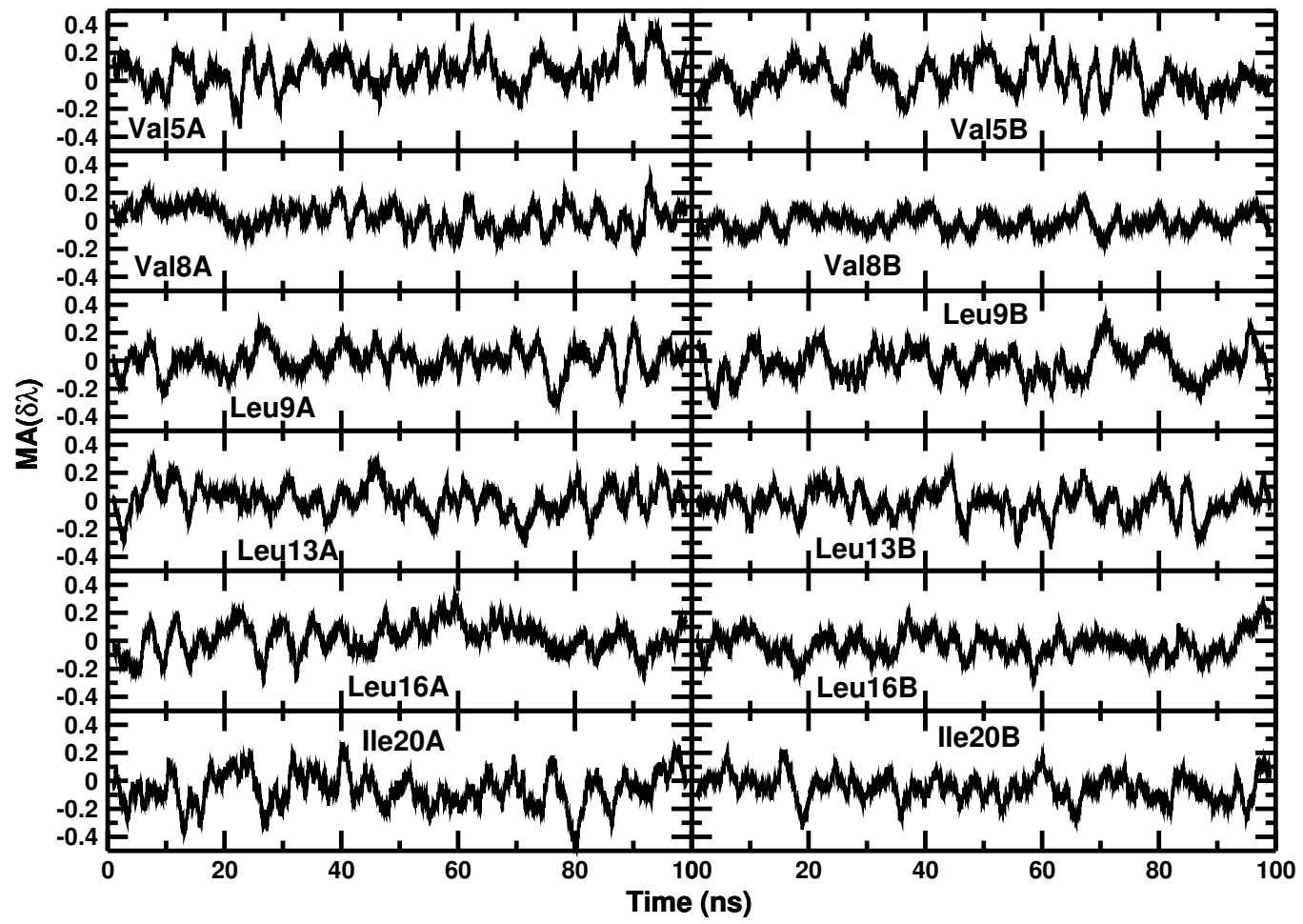

Figure 10: Difference in hydrophobicity for the residues in the melittin dimer hydrophobic pocket as defined in Ref.7 for the rigid simulations in the 51 and $60 \AA$ boxes. They are residues Val5, Val8, Leu13, Leu16, Ile20. The data reported is $\Delta \delta \lambda=\delta \lambda_{60}-\delta \lambda_{51}$, i.e. the change in LH from the simulation in the two water boxes. The maximum instantaneous change in $\Delta \delta \lambda_{\text {phob }}$ due to the box size is $40 \%$; most differences are $20 \%$ or less.

It is also of interest to compare the difference in hydrophobicity for simulations of rigid melit- 
tin in the two water boxes because all differences must arise from the size of the water box. Figure 10 shows the difference between the $51 \AA$ and the $60 \AA$ boxes in LH of the residues in the hydrophobic pocket. The average fluctuations are of the order of 0.1 units with maximum differences of 0.4 units. The difference between simulations with rigid and flexible melittin can also be seen when comparing the radial distribution functions, $g(r)$, between $\mathrm{C}_{\alpha}$ atoms of selected residues and water and the corresponding water occupations $N(r)$ (see Figures S19 and S20). The residues were chosen in accord with the results from Table 3 . For example, in the $51 \AA$ box for rigid melittin the values for Val5A and Val5B are $\delta \lambda_{\text {phob }}=1.19$ and 1.48, respectively, which change to 1.25 and 1.49 in the larger $60 \AA$ box; i.e., this is a change of $5 \%$ at most. Figure S19A shows that $g(r)$ for Val5A and Val5B are very similar for both water box sizes. This suggests that the difference of $\sim 0.25$ in Table 3 for the two water boxes must arise from the orientation of the water molecules within the $6 \AA$ cut-off.

Conversely, for flexible melittin the differences for LH in the two water boxes can be substantial. As an example, Leu9B $\delta \lambda_{\text {phob }}^{51 \mathrm{~A}}=0.81$ is compared with $\delta \lambda_{\text {phob }}^{60 \mathrm{~A}}=1.07$ for the two box sizes. This is also evident from Figure S20A and B (right panel) for which $g(r)$ and $N(r)$ have increased amplitudes for the larger water box. For Val5B, $\lambda_{\text {phob }}^{51 \mathrm{~A}}=1.05$ is larger in the smaller box than $\lambda_{\text {phob }}^{60 \mathrm{~A}}=0.86$, whereas the amplitude of $g(r)$ up to the $6 \AA$ cut-off in the larger box is larger than that in the smaller box. Hence, the difference found in the two box sizes must arise from the angular orientations of the water molecules relative to the protein surface. These analyses suggest that both the distance-dependence (reflected in $g(r)$ and $N(r)$ ) and the angular orientation, as measured by $\lambda_{\text {phob }}$, can depend on box size and potentially influence the thermodynamic stability of the two proteins.

The average hydrophobicity for each residue during the simulation is reported in Figure 11A (black for chain A and red for chain B). The decreased hydrophobicity of the central part of the chains (from Val5 to Leu16) is highlighted by the lower $\delta \lambda_{\text {phob }}$ (of 0.1-0.2 units) compared 


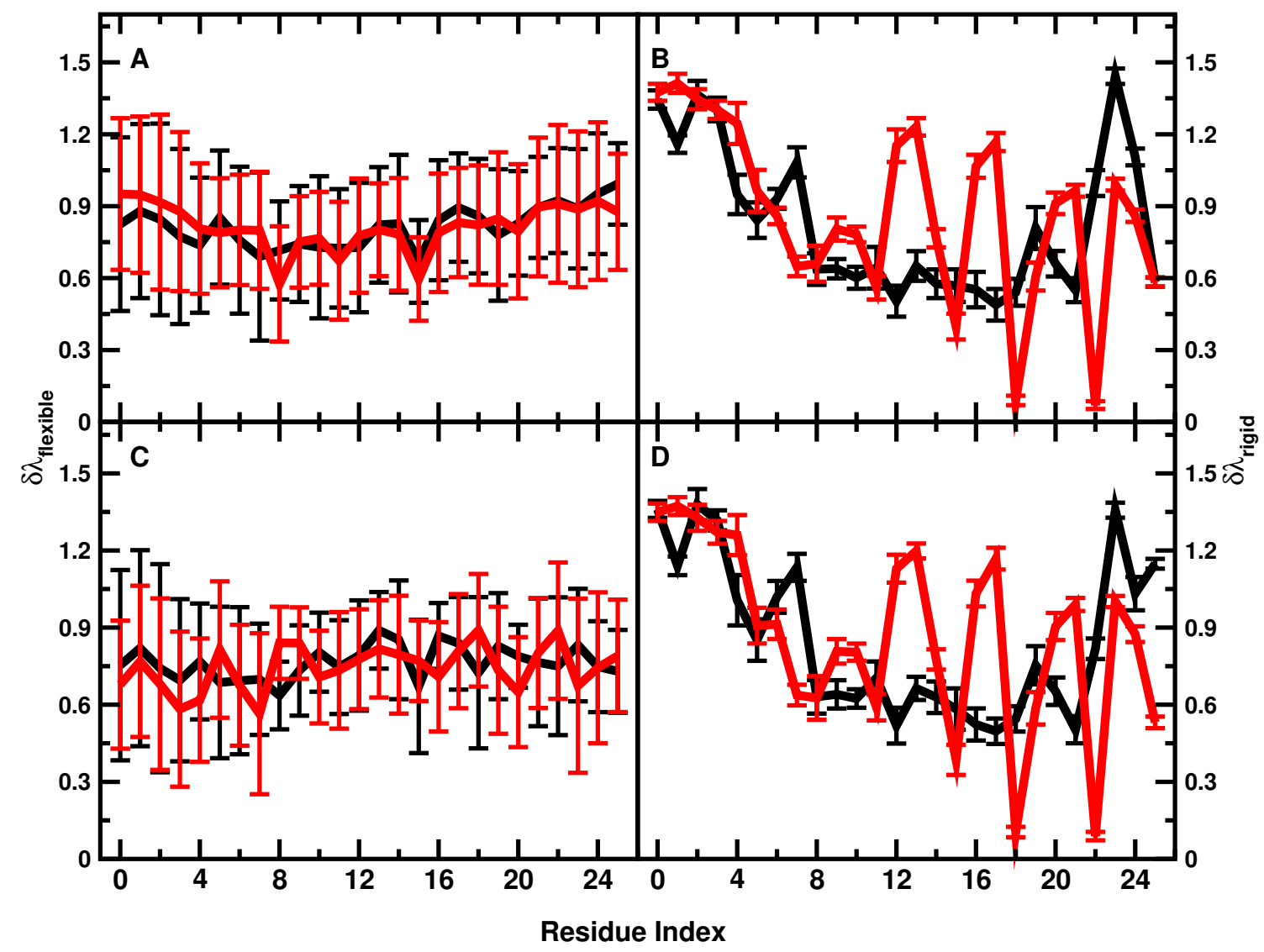

Figure 11: Average $\delta \lambda_{\text {phob }}$ per residue for the 100 ns simulation for chains A (black) and B (red) for the flexible (panels $\mathrm{A}$ and $\mathrm{C}$ ) and the rigid (panels $\mathrm{B}$ and $\mathrm{D}$ ) melittin dimer. The top row is for the $51 \AA$ box and the bottom row for the $60 \AA$ box. The value of the $\mathrm{LH}$ ranges from $0.50<\delta \lambda_{\text {phob }}<1.00$ for the flexible dimer and between $0.00<\delta \lambda_{\text {phob }}<1.50$ for the rigid dimer. For the rigid dimer, the central part of the A-helix has consistently lower values of LH compared with the $\mathrm{C}$ - and N-terminal parts. For chain B larger variations in LH are found for some of the residues due to its different structure. The periodicity of the red traces (panels B and D) reflects the helical structure which is apparent for monomer B but less so for monomer A. For flexible melittin the variation of LH along the sequence is much smoother than for rigid melittin as a consequence of dynamical averaging. 
with the C- and N-terminal parts. The main difference between the simulation results for the flexible (left) and rigid (right) melittin dimer is the amplitude of the fluctuation of the hydrophobicity, but not its sign. Simulations of rigid melittin in the $51 \AA$ (panels A and B) and $60 \AA$ boxes (panels $\mathrm{C}$ and D) are similar to one another but they differ along the trajectory by up to $\Delta \delta \lambda \sim 0.4$ (see Figure 10). There are also differences between the A (black) and B chains (red) for rigid melittin. The difference in the monomer structures (RMSD of $1.6 \AA$ ) leads to significant differences in $\delta \lambda$ (see Figures 11 A and B. As an example, Leu13A is considerably more hydrophobic $\left(\delta \lambda_{\text {Leu13A }}=0.7\right)$ than Leu13B $\left(\delta \lambda_{\text {Leu13B }}=1.4\right)$. Inspection of the dimer structure shows that Leu13A points toward the dimerization interface whereas Leu13B points away from it into the solvent. For flexible melittin (panels A and C), the LH for the A and B chains are more similar to one another for both box sizes. This is a consequence of averaging along the structural dynamics. It also suggests that simulations on the 100 ns time scale are sufficient to converge $\delta \lambda$ for melittin. Nevertheless, there remain certain differences between simulations in the two water boxes for individual residues, e.g. $\left\langle\delta \lambda_{\text {Val5B }}^{51}\right\rangle=1.05$ vs. $\left\langle\delta \lambda_{\text {Val5B }}^{60}\right\rangle=0.86$, see Figure 11 and Table 3.

Table 3: Average $\delta \lambda_{\text {phob }}$ for the hydrophobic residues from Figure 1 in Ref. ${ }^{7}$ The time series are shown in Figure S17

\begin{tabular}{l|r|r|r|r} 
Residue & $51 \AA$ flexible & $51 \AA$ rigid & $60 \AA$ flexible & $60 \AA$ rigid \\
\hline Val5A & 0.976 & 1.185 & 1.003 & 1.246 \\
Val8A & 0.929 & 1.322 & 0.941 & 1.375 \\
Leu9A & 0.954 & 0.875 & 0.889 & 0.876 \\
Leu13A & 0.967 & 0.741 & 1.030 & 0.756 \\
Leu16A & 0.909 & 0.808 & 0.917 & 0.824 \\
Ile20A & 1.019 & 1.049 & 1.071 & 0.992 \\
Val5B & 1.046 & 1.484 & 0.861 & 1.494 \\
Val8B & 1.038 & 0.889 & 0.806 & 0.877 \\
Leu9B & 0.814 & 0.900 & 1.071 & 0.867 \\
Leu13B & 1.016 & 1.393 & 1.006 & 1.373 \\
Leu16B & 0.835 & 0.637 & 1.012 & 0.626 \\
Ile20B & 1.087 & 0.845 & 0.988 & 0.824
\end{tabular}

Finally, the two-dimensional LH-CCM (see Figure S21) for all four systems and their differ- 
ences between rigid and flexible dimer (bottom row of Figure S21) have been determined. As can be anticipated from Figure 10, the LH cross correlation maps for the two box sizes are similar for rigid melittin dimer (see Figure S22 left panel). On the other hand, the differences between rigid and flexible melittin dimer in the two boxes are considerably larger, as the bottom row of Figure S21 demonstrates. While for the $51 \AA$ box differences primarily occur at the interface (upper left quadrant), differences for the larger $60 \AA$ box occur both at the interface and along the two helices. Furthermore, the amplitude of the differences increases in going from the $51 \AA$ to the $60 \AA$ box.

The difference between flexible melittin dimer in the $51 \AA$ and $60 \AA$ boxes is shown in Figure S22, right panel. Increasing the box size leads to more pronounced cross correlation peaks between the beginning of helix A and the end of helix B. A slightly less pronounced increase in the correlation is found for the end of chain A and the beginning of chain B. Corresponding radial distribution functions are reported in Figure S23. Even for rigid melittin in the $51 \AA$ (red) and $60 \AA$ (blue) boxes (e.g. for Gly3A and Gln26B) there are slight differences between the $g(r)$. Compared with rigid melittin, the $g(r)$ for flexible melittin are all less structured. Except for Ile2B they also agree well for the two box sizes.

For the rigid and flexible dimer, differences for the two box sizes also occur as shown in the $\triangle \mathrm{CCC}$ map (Figure S22). They effect the local hydrophobicity which is computed from the water structuring. For the rigid protein surface the differences are essentially independent of box size, as shown in the left panel. For the flexible dimer there are significant differences. They arise both from protein structural changes and the surrounding water structuring. In this context it is interesting to note that $g(r)$ around Leu9A and Leu9B in Figure S20 in the $60 \AA$ box are virtually identical, whereas the LH differs by almost $15 \%$ (0.89 vs. 1.07 , see Table 3). As LH includes both the distance between the water oxygen atom relative to the protein surface and the angular orientation of the $\mathrm{OH}$ vector, this difference in $\mathrm{LH}$ 
is likely to be related to different orientations of the water network around Leu9A and Leu9B.

\section{Conclusions}

The present work analyzed the local hydrophobicity around key residues at the protein interfaces for hemoglobin and melittin. It was found that the local hydrophobicity measure for $\mathrm{Hb}$ provides valuable insight into the effect of different box sizes from MD simulations. Specifically, analysis of the local hydrophobicity cross correlation coefficients for Hb provided a dynamical view of Perutz's stereochemical model involving breaking and formation of salt bridges at the $\alpha_{1} / \beta_{2}$ and $\alpha_{2} / \beta_{1}$ interfaces. Also, the more detailed analysis of the simulations in the $90 \AA$ and $120 \AA$ boxes demonstrates that they decay to known but different intermediate structures upon destabilization of the $\alpha / \beta$ interface following a decrease in LH, i.e. as a consequence of reduced water density or change of water orientation at the protein/water interface. This is consistent with earlier findings ${ }^{4}$ that reported a reduced number of water-water hydrogen bonds for the smaller boxes, which influences the equilibrium between water-water and water-protein contacts and hence the water activity. The present results also support recent extensive simulation studies of the $\mathrm{A} \beta$ peptide which show that the hydrophobic surface area increases significantly in small cells along with the standard deviation in exposure and backbone conformations. ${ }^{29}$ As is also reported here (see Figure 7), hydrophilic exposure was found to dominate in large boxes whereas hydrophobic exposure is prevalent in small cells. This suggests there is a weakening of the hydrophobic effect in smaller water box sizes.

Early experiments indicate that $\mathrm{T}_{0}$ is significantly $\left(\sim 8 \mathrm{kcal} / \mathrm{mol}\right.$, equivalent to $K_{\frac{T_{0}}{R_{0}}}=$ $6.7 \times 10^{5}$ ) more stable than $\mathrm{R}_{0} \cdot{ }^{1}$ Also, the rate for the $\mathrm{R}_{0} \rightarrow \mathrm{T}_{0}$ has been determined as $15700 \pm 700 \mathrm{~s}^{-1}$ at $303 \mathrm{~K}$, corresponding to a transition time of $\sim 20 \mu \mathrm{s} . \underline{30}$ As shown in 
Ref. $\frac{31}{3}$ this implies that the $\mathrm{T}_{0} \rightarrow \mathrm{R}_{0}$ transition occurs on a time scale of 1 to $10 \mathrm{~s}$ by use of the Arrhenius equation. This is far too long to be sampled directly by MD simulations with explicit solvent in a statistically meaningful way. As an example, for association free energies in protein-ligand and protein-protein interactions from replica exchange coarse grained simulations, a total simulation time of $>5 \mu$ s was deemed necessary for convergence ${ }^{32}$ and similar studies were carried out for protein-ligand interactions using atomistic force fields. ${ }^{33[34} \mathrm{Al}-$ ternative approaches, such as conjugate peak refinement, string methods, or nudged elastic band in explicit solvent are also ways to more quantitatively investigate the transition state region. $35+37$ However, to quantify differences between the $T_{0}$ and $R_{0}$ structure or structures evolving towards the $\mathrm{R}_{0}$ state (as done here), explicit knowledge of the transition state region is not required.

For the melittin dimer the role of box size on the hydration dynamics was expected to be smaller, based on earlier work on the hydrophobic effect. ${ }^{38}$ Nevertheless, the analysis of rigid melittin dimer, which was studied in previous work, ${ }^{7}$ suggests that the water distribution is affected by the box size for the $51 \AA$ and $60 \AA$ boxes. These differences become more pronounced when the protein structure is allowed to change in the simulations.

Complementary to radial distribution functions $g(r)$, the local hydrophobicity (LH) provides a time-dependent quantitative local measure characterizing the water dynamics and structure around a protein. When combined with time-dependent structural information a more complete picture for the coupled protein-water dynamics emerges. It provides valuable information about thermodynamic manifestations of structural changes at a molecular level. 


\section{Data and Code Availability}

The water-structure analysis code used to calculate $\delta \lambda_{\text {phob }}$ is publicly available at https: //github.com/mjmn/interfacial-water-structure-code.

\section{Acknowledgment}

Support by the Swiss National Science Foundation through grants 200021-117810, the NCCR MUST (to MM), and the University of Basel is acknowledged. The support of MK by the CHARMM Development Project is gratefully acknowledged. AW, MN, and SS were supported by the National Science Foundation under CHE-1654415

\section{References}

(1) Edelstein, S. Extensions of allosteric model for haemoglobin. Nature 1971, 230, 224227.

(2) Hub, J. S.; Kubitzki, M. B.; de Groot, B. L. Spontaneous Quaternary and Tertiary T-R Transitions of Human Hemoglobin in Molecular Dynamics Simulation. PLoS Comput Biol 2010, 6, e1000774.

(3) Yusuff, O. K.; Babalola, J. O.; Bussi, G.; Raugei, S. Role of the Subunit Interactions in the Conformational Transitions in Adult Human Hemoglobin: An Explicit Solvent Molecular Dynamics Study. J. Phys. Chem. B 2012, 116, 11004-11009.

(4) El Hage, K.; Hedin, F.; Gupta, P. K.; Meuwly, M.; Karplus, M. Valid molecular dynamics simulations of human hemoglobin require a surprisingly large box size. eLife 2018, 7, e35560. 
(5) Gapsys, V.; de Groot, B. L. Comment on 'Valid molecular dynamics simulations of human hemoglobin require a surprisingly large box size'. eLife 2019, 8, e44718.

(6) El Hage, K.; Hedin, F.; Gupta, P. K.; Meuwly, M.; Karplus, M. Response to comment on 'Valid molecular dynamics simulations of human hemoglobin require a surprisingly large box size'. eLife 2019, 8, e45318.

(7) Cheng, Y.; Rossky, P. Surface topography dependence of biomolecular hydrophobic hydration. Nature 1998, 392, 696-699.

(8) Terwilliger, T. C.; Eisenberg, D. The structure of melittin. I. Structure determination and partial refinement. J. Biol. Chem. 1982, 25\%, 6010-6015.

(9) Terwilliger, T. C.; Eisenberg, D. The structure of melittin. J. Biol. Chem. 1982, 257, L6015.

(10) Shin, S.; Willard, A. P. Characterizing Hydration Properties Based on the Orientational Structure of Interfacial Water Molecules. J. Chem. Theor. Comput. 2018, 14, 461-465.

(11) Brooks, B. R. et al. CHARMM: The Biomolecular Simulation Program. J. Comput. Chem. 2009, 30, 1545-1614.

(12) Best, R. B.; Zhu, X.; Shim, J.; Lopes, P. E. M.; Mittal, J.; Feig, M.; MacKerell, A. D., Jr. Optimization of the Additive CHARMM All-Atom Protein Force Field Targeting Improved Sampling of the Backbone phi, psi and Side-Chain chi(1) and chi(2) Dihedral Angles. J. Chem. Theor. Comput. 2012, 8, 3257-3273.

(13) Anderson, D.; Terwilliger, T. C.; Wickner, W.; Eisenberg, D. Melittin forms crystals which are suitable for high resolution X-ray structural analysis and which reveal a molecular 2-fold axis of symmetry. J. Biol. Chem. 1980, 255, 2578-2582.

(14) Essmann, U.; Perera, L.; Berkowitz, M. L.; Darden, T.; Lee, H.; Pedersen, L. G. A smooth particle mesh Ewald method. J. Chem. Phys. 1995, 103, 8577-8593. 
(15) Huang, J.; Rauscher, S.; Nawrocki, G.; Ran, T.; Feig, M.; de Groot, B. L.; Grubmueller, H.; MacKerell, A. D., Jr. CHARMM36m: an improved force field for folded and intrinsically disordered proteins. Nat. Meth. 2017, 14, 71-73.

(16) Swope, W.; Anderson, H.; Berens, P.; Wilson, K. A Copmuter-Simulation Method for the Calculation of Equilibrium-Constants for the Formation of Physical Clusters of Molecules - Application to Small Water Clusters. J. Chem. Phys. 1982, 76, 637-649.

(17) Hoover, W. G. Canonical dynamics: Equilibrium phase-space distributions. Phys. Rev. A 1985, 31, 1695-1697.

(18) van Gunsteren, W.; Berendsen, H. Algorithms for macromolecular dynamics and constraint dynamics. Mol. Phys. 1977, 34, 1311-1327.

(19) Shin, S.; Willard, A. P. Water's Interfacial Hydrogen Bonding Structure Reveals the Effective Strength of Surface-Water Interactions. J. Phys. Chem. B 2018, 122, 67816789.

(20) Willard, A. P.; Chandler, D. Instantaneous Liquid Interfaces. J. Phys. Chem. B 2010, $114,1954-1958$.

(21) Perutz, M. F. Stereochemistry of Cooperative Effects in Haemoglobin: HaemHaem Interaction and the Problem of Allostery. Nature 1970, 228, 726-734.

(22) Safo, M. K.; Abraham, D. J. The Enigma of the Liganded Hemoglobin End State: A Novel Quaternary Structure of Human Carbonmonoxy Hemoglobin. Biochem. 2005, $44,8347-8359$.

(23) Park, S.-Y.; Yokoyama, T.; Shibayama, N.; Shiro, Y.; Tame, J. R. H. 1.25 angstrom resolution crystal structures of human haemoglobin in the oxy, deoxy and carbonmonoxy forms. J. Mol. Biol. 2006, 360, 690-701. 
(24) Ichiye, T.; Karplus, M. Collective motions in proteins: A covariance analysis of atomic fluctuations in molecular dynamics and normal mode simulations. Prot. Struct. Funct. Genom. 1991, 11, 205-217.

(25) Karplus, M.; Ichiye, T. Fluctuation and cross correlation analysis of protein motions observed in nanosecond molecular dynamics simulations. J. Mol. Biol. 1996, 263, 120122.

(26) Desmond, J. L.; Koner, D.; Meuwly, M. Probing the Differential Dynamics of the Monomeric and Dimeric Insulin from Amide-I IR Spectroscopy. J. Phys. Chem. B 2019, 123, 6588-6598.

(27) Cutfield, J.; Cutfield, S.; Dodson, E.; Dodson, G.; Emdin, S.; Reynolds, C. Structure and biological activity of hagfish insulin. J. Mol. Biol. 1979, 132, 85-100.

(28) Baker, E. N.; Blundell, T. L.; Cutfield, J. F.; Dodson, E. J.; Dodson, G. G.; Hodgkin, D. M. C.; Hubbard, R. E.; Isaacs, N. W.; Reynolds, C. D.; Sakabe, K.; Sakabe, N.; Vijayan, N. M. The structure of 2 Zn pig insulin crystals at $1.5 \AA$; resolution. Philosophical Transactions of the Royal Society of London. B, Biological Sciences 1988, 319, 369456.

(29) Mehra, R.; Kepp, K. P. Cell size effects in the molecular dynamics of the intrinsically disordered A beta peptide. J. Chem. Phys. 2019, 151.

(30) Sawicki, C.; Gibson, Q. Quaternary conformational changes in human hemoglobin studied by laser photolysis of carboxyhemoglobin. J. Biol. Chem. 1976, 251, 1533-1542.

(31) Cui, Q.; Karplus, M. Allostery and cooperativity revisited. Prot. Sci. 2008, 17, 12951307.

(32) Domanski, J.; Hedger, G.; Best, R. B.; Stansfeld, P. J.; Sansom, M. S. P. Conver- 
gence and Sampling in Determining Free Energy Landscapes for Membrane Protein Association. J. Phys. Chem. B 2017, 121, 3364-3375.

(33) El Hage, K.; Mondal, P.; Meuwly, M. Free energy simulations for protein ligand binding and stability. Mol. Sim. 2018, 44, 1044-1061.

(34) Ngo, S. T.; Vu, K. B.; Bui, L. M.; Vu, V. V. Effective Estimation of Ligand-Binding Affinity Using Biased Sampling Method. ACS OMEGA 2019, 4, 3887-3893.

(35) Fischer, S.; Olsen, K. W.; Nam, K.; Karplus, M. Unsuspected pathway of the allosteric transition in hemoglobin. Proc. Natl. Acad. Sci. 2011, 108, 5608-5613.

(36) Ovchinnikov, V.; Karplus, M.; Vanden-Eijnden, E. Free energy of conformational transition paths in biomolecules: The string method and its application to myosin VI. $J$. Chem. Phys. 2011, 134.

(37) Henkelman, G.; Uberuaga, B.; Jonsson, H. A climbing image nudged elastic band method for finding saddle points and minimum energy paths. J. Chem. Phys. 2000, 113, 9901-9904.

(38) Chandler, D. Interfaces and the driving force of hydrophobic assembly. Nature 2005, 437, 640-647. 